03

\title{
Об излучении поверхностного источника по нормали к границе полупространства и плоскослоистой структуры
}

\author{
(C) А.Б. Петрин \\ Объединенный институт высоких температур РАН, \\ 125412 Москва, Россия \\ e-mail: a_petrin@mail.ru \\ Поступила в редакцию 09.03.2021 г. \\ В окончательной редакции 09.03.2021 г. \\ Принята к публикации 22.03.2021 г.
}

На основе строгой теории излучения элементарного источника тока, расположенного на границе плоскослоистой структуры, рассмотрено излучение произвольного распределения поверхностного тока. Получено строгое соотношение между излучаемыми по нормали полями, порожденными произвольным распределением стороннего поверхностного тока в полупространства, окружающие плоскослоистую структуру. Показано, что отношение амплитуд электрических полей на одном и том же расстоянии от источника, излучающего в противоположных направлениях, нормально к плоскослоистой структуре, определяется простым алгебраическим выражением, включающим электромагнитные параметры сред и толщины пленок структуры. Рассмотрены частные случаи поверхностного источника излучения на границе одной однородной пленки и поверхностного источника на границе двух однородных полупространств.

Ключевые слова: наноантенны, оптические сенсоры.

DOI: $10.21883 /$ OS.2021.07.51082.1986-21

\section{Введение}

Теоретические методы нахождения излучения от элементарного точечного электрического диполя, расположенного на плоской границе двух сред, были развиты на заре эры использования электромагнитных волн для передачи сигналов вдоль земной поверхности [1]. В дальнейшем, эти методы получили дальнейшее развитие в связи с возникающими новыми задачами радиосвязи $[2,3]$. Развитие антенной техники привело к развитию теории излучения элементарного диполя, расположенного на границе плоскослоистой среды и теории излучения микрополосковых антенн [4-6], которые являются наилучшими конструкциями, с точки зрения их совместимости с микрополосковыми интегральными схемами, которые играют особую роль в современной технике, так как могут быть изготовлены из фольгированных материалов высокопроизводительными методами печати и травления.

Существующие теоретические методы расчета излучения антенн, расположенных на границе плоскослоистых структур сложны (описываются в терминах диадных функций Грина [7]). Кроме того, сама теория распространения волн в слоистых структурах содержит много тонких моментов, таких как необходимость правильного выбора ветвей аналитических функций при записи волн в слоях, которые могут приводить к ошибочным выводам [8]. В работах [9-11] был предложен оригинальный вариант строгой электромагнитной теории излучения элементарного тока, расположенного на границе или внутри плоскослоистой структуры. Так, для случая излучения элементарного диполя, расположенного на сво- бодной границе одной пленки, был продемонстрирован метод аналитического упрощения решения, имеющий, как кажется, потенциально общетеоретическое значение. Этот метод позволил привести формулы для излучаемых полей к одномерным интегралам, что существенно упростило анализ задачи и ускорило численные расчеты, а в случае нахождения полей в дальней зоне, позволило вообще избежать интегрирования [12]. Ниже, указанный метод применен для установления общих закономерностей излучения произвольных сторонних поверхностных токов, расположенных на границе плоскослоистой структуры. Нахождение таких общих закономерностей является важным для конструирования и нахождения характеристик излучения наноантенн, которые используются для визуализации живых клеток, манипулирования наночастицами, зондирования и повышения эффективности солнечных элементов [13-15].

\section{Постановка задачи. Излучение элементарного излучателя, расположенного внутри плоскослоистой структуры}

Рассмотрим задачу излучения электромагнитной волны источником тока, изменяющимся во времени гармонически с циклической частотой $\omega$. Пусть этот источник расположен внутри плоской слоистой структуры, состоящей из нескольких пленок, и из, окружающих слоистую структуру, двух полупространств. Для определенности сначала будем считать, что источник расположен в 


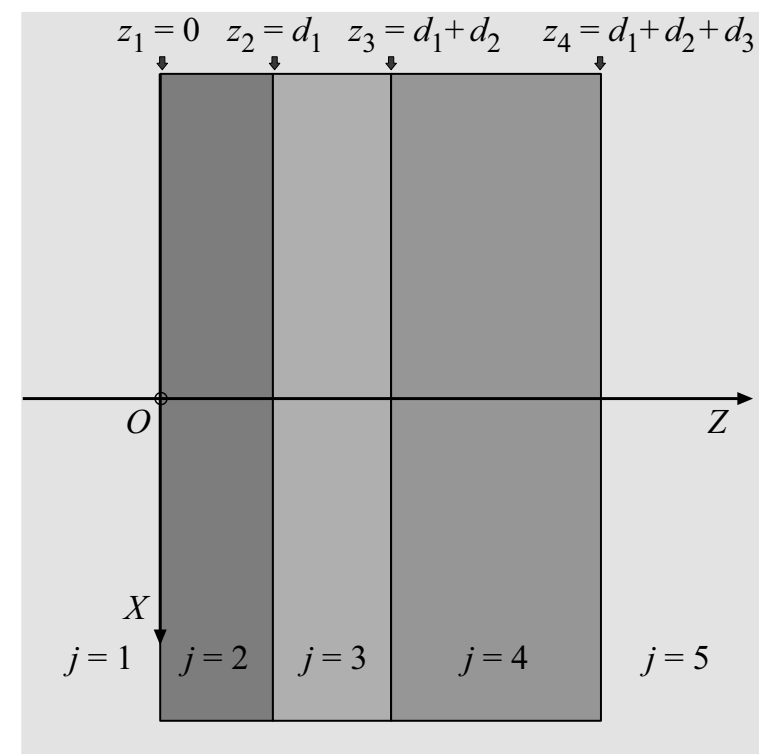

Pис. 1. Геометрия плоскослоистой структуры, состоящей из трех пленок.

одной из пленок, а затем обобщим эту задачу на случай, когда источник расположен на границе между слоями.

Пусть общее число пленок равно $N_{f}$, толщина $m$-й пленки равна $d_{m}$ и полная толщина слоистой структуры равна $d_{\text {tot }}=\sum_{m=1}^{N_{f}} d_{m}$. Общее число границ между пленками обозначим как $N=N_{f}+1$. Пронумеруем области пространства $j=1, \ldots,(N+1)$ (рис. 1 показана для примера задача с $N=4$ и $\left.N_{f}=3\right)$. Предположим, что пленки имеют абсолютные комплексные диэлектрические и магнитные проницаемости равные $\varepsilon_{j}$ и $\mu_{j}$ на рассматриваемой частоте $\omega$, а снаружи такой плоскослоистой структуры находятся однородные полупространства с проницаемостями $\varepsilon_{1}, \mu_{1}$ и $\varepsilon_{N+1}, \mu_{N+1}$ (свободное пространство). Обозначим также через $z_{j}-$ координаты $N$ границ пленок по оси $Z$ следующим образом: $z_{1}=0$, $z_{j}=\sum_{m=1}^{j-1} d_{m}$ при $j_{2}, \ldots,(N+1)$.

Уравнения Максвелла для области с номером $j$ можно записать в виде:

$$
\begin{gathered}
\operatorname{rot} \mathbf{E}_{j}=i \omega \mathbf{B}_{j}, \\
\operatorname{rot} \mathbf{B}_{j}=\mu_{j}\left(-i \omega \varepsilon_{j} \mathbf{E}_{j}+\mathbf{J}_{j}\right),
\end{gathered}
$$

где $\mathbf{E}_{j}, \mathbf{B}_{j}$ и $\mathbf{J}_{j}-$ векторы напряженности электрического поля, индукция магнитного поля и плотность стороннего (известного) тока в области с номером $j$ (если источник тока отсутствует в области $j$, то $\mathbf{J}_{j}=0$ ). Предполагается комплексное временное представление в виде $e^{-i \omega t}$. Кроме того, в данной работе будем считать, что сторонние токи направлены в одной плоскости, параллельной границе пленок ( $\mathbf{J}_{j}$ имеет только $x$ и $y$ составляющие).
Решая уравнения Максвелла в каждой области задачи, найдем электромагнитное поле во всех областях. Рассмотрим сначала следующую вспомогательную задачу.

\section{Распространение электромагнитной волны в слое, свободном от сторонних токов}

Пусть в области с номером $j$ нет сторонних токов между границами $z_{j-1}$ и $z_{j}$ (рис. 2), а диэлектрическая и магнитная проницаемости однородной среды пленки равны $\varepsilon_{j}$ и $\mu_{j}$ соответственно. Из уравнений (1) и (2) получаем уравнение для электрического поля

$$
\operatorname{rot} \operatorname{rot} E_{j}-\omega^{2} \varepsilon_{j} \mu_{j} E_{j}=0
$$

и такое же уравнение для поля $\mathbf{B}_{j}$. В рассматриваемой области $\operatorname{div} \mathbf{E}_{j}=0$ и $\operatorname{div} \mathbf{B}_{j}=0$, тогда, используя оператор Лапласа $\Delta=\partial_{x x}^{2}+\partial_{y y}^{2}+\partial_{z z}^{2}$ и учитывая векторное тождество $\operatorname{rot} \operatorname{rot} \mathbf{F}=\operatorname{grad} \operatorname{div} \mathbf{F}-\Delta \mathbf{F}$, из (3) получим

$$
\Delta \mathbf{E}_{j}+\omega^{2} \varepsilon_{j} \mu_{j} \mathbf{E}_{j}=0,
$$

и такое же уравнение для поля $\mathbf{B}_{j}$.

Подставим в полученные выше уравнения компоненты полей в виде двумерных фурье-разложений. Например, представление для $x$-компоненты электрического поля представим в виде $E_{j, x}(x, y, z)=$ $=(2 \pi)^{-2} \int_{-\infty}^{+\infty} \int_{-\infty}^{+\infty} \tilde{E}_{j, x}(\xi, \eta, z) e^{i(\xi x+\eta y)} d \xi d \eta, \quad$ где фурьеобраз определяется выражением $\tilde{E}_{j, x}(\xi, \eta, z)=$ $=\int_{-\infty}^{+\infty} \int_{-\infty}^{+\infty} E_{j, x}(x, y, z) e^{-i(\xi x+\eta y)} d x d y$. Для остальных компонент полей будем использовать аналогичные

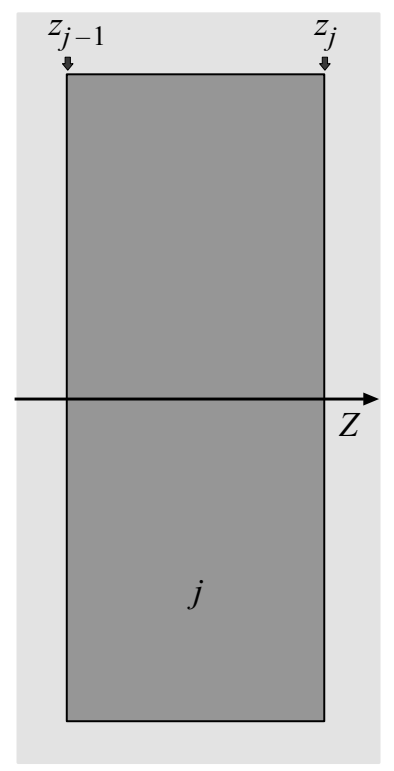

Рис. 2. Пленка с номером $j$, расположенная между границами $z_{j-1}$ и $z_{j}$. 
представления и соответствующие символы. Переходя к фурье-образам, получаем из векторного уравнения (4) три уравнения

$$
\begin{gathered}
\frac{d^{2} \tilde{E}_{j, x}}{d z^{2}}+\gamma_{j}^{2} \tilde{E}_{j, x}=0 ; \quad \frac{d^{2} \tilde{E}_{j, y}}{d z^{2}}+\gamma_{j}^{2} \tilde{E}_{j, y}=0 ; \\
\frac{d^{2} \tilde{E}_{j, z}}{d z^{2}}+\gamma_{j}^{2} \tilde{E}_{j, z}=0,
\end{gathered}
$$

где $\gamma_{j}=\sqrt{k_{j}^{2}-\xi^{2}-\eta^{2}}, k_{j}=\omega \sqrt{\mu_{j} \varepsilon_{j}}$.

Общие решения уравнений (5), для направлений распространения волн вдоль „,+“ и против „-“ оси $Z$, можно записать в виде

$$
\mathbf{E}_{j}^{ \pm}(x, y, z)=\frac{1}{(2 \pi)^{2}} \int_{-\infty}^{+\infty} \int_{-\infty}^{+\infty}\left(\begin{array}{c}
\tilde{E}_{j, x}^{ \pm} \\
\tilde{E}_{j, y}^{ \pm} \\
\tilde{E}_{j, z}^{ \pm}
\end{array}\right) e^{ \pm i \gamma_{j} z} e^{i(\xi x+\eta y)} d \xi d \eta,
$$

или, учитывая $\operatorname{div} \mathbf{E}_{j}=0 \quad$ и, следовательно $\xi \hat{E}_{j, x}^{ \pm}+\eta \hat{E}_{j, y}^{ \pm} \pm \gamma_{j} \hat{E}_{j, z}^{ \pm}=0$, в виде

$$
\begin{aligned}
\mathbf{E}_{j}^{ \pm}(x, y, z)= & \frac{1}{(2 \pi)^{2}} \int_{-\infty}^{+\infty} \int_{-\infty}^{+\infty}\left(\begin{array}{cc}
1 & 0 \\
0 & 1 \\
\mp \xi / \gamma_{j} & \mp \eta / \gamma_{j}
\end{array}\right)\left(\begin{array}{c}
\hat{E}_{j, x}^{ \pm} \\
\hat{E}_{j, y}^{ \pm}
\end{array}\right) \\
& \times e^{ \pm i \gamma_{j} z} e^{i(\xi x+\eta y)} d \xi d \eta
\end{aligned}
$$

Поэтому общие решения уравнений (5) в области $\left[z_{j-1}, z_{j}\right]$ можно записать в виде

$$
\begin{aligned}
& \mathbf{E}_{j}(x, y, z)=\frac{1}{(2 \pi)^{2}} \int_{-\infty}^{+\infty} \int_{-\infty}^{+\infty}\left(\begin{array}{cc}
1 & 0 \\
0 & 1 \\
-\xi / \gamma_{j} & -\eta / \gamma_{j}
\end{array}\right)\left(\begin{array}{c}
\hat{E}_{j, x}^{+} \\
\hat{E}_{j, y}^{+}
\end{array}\right) \\
& \quad \times e^{i \gamma_{j}\left(z-z_{j-1}\right)} e^{i(\xi x+\eta y)} d \xi d \eta \\
& \quad+\frac{1}{(2 \pi)^{2}} \int_{-\infty}^{+\infty} \int_{-\infty}^{+\infty}\left(\begin{array}{cc}
1 & 0 \\
0 & 1 \\
\xi / \gamma_{j} & \eta / \gamma_{j}
\end{array}\right)\left(\begin{array}{c}
\hat{E}_{j, x}^{-} \\
\hat{E}_{j, y}^{-}
\end{array}\right) \\
& \quad \times e^{-i \gamma_{j}\left(z-z_{j}\right)} e^{i(\xi x+\eta y)} d \xi d \eta
\end{aligned}
$$

Обратим внимание на отличие в форме записи (6) и (7). Формально эти уравнения переходят одно в другое, они описывают волны, распространяющиеся в противоположных направлениях по оси $Z$. Однако, формулы содержат функции $\gamma_{j}=\sqrt{k_{j}^{2}-\xi^{2}-\eta^{2}}$. Для однозначного определения вида записи решений необходимо однозначно выбрать аналитическую ветвь функции комплексного переменного $\gamma_{j}(\lambda)$, где $\lambda^{2}=\xi^{2}+\eta^{2}$.

Как правило, для обычных сред без поглощения используют ветвь [3]

$$
\gamma_{j}(\lambda)= \begin{cases}\sqrt{k_{j}^{2}-\lambda^{2}}, & \lambda^{2} \leq k_{j}^{2} \\ i \sqrt{\lambda^{2}-k_{j}^{2}}, & \lambda^{2} \geq k_{j}^{2}\end{cases}
$$

Если вместо (8) взять другую аналитическую ветвь, с отрицательной мнимой зависимостью, то волны с большими $\lambda$ будут экспоненциально возрастать $\mathrm{c} z$ при удалении от источников полей, что противоречит принципу причинности.

Более строго, в случае поглощающей среды, существует две точки ветвления функции $\gamma_{j}(\lambda)$ : точка $k_{j, 1}=\omega \sqrt{\left|\varepsilon_{j}\right|\left|\mu_{j}\right|} \exp \left(i\left(\arg \left(\varepsilon_{j}\right)+\arg \left(\mu_{j}\right)\right) / 2\right)$ и точка $k_{j, 2}=e^{i \pi} k_{j, 1}$. Аналитическую ветвь функции $\gamma_{j}(\lambda)$ пригодную, в том числе, для описания материалов с отрицательным преломлением [16] и переходящую в (8) для обычной непоглощающей среды, можно определить как

$$
\begin{aligned}
\gamma_{j}(\lambda)= & \sqrt{\left|k_{j, 1}-\lambda\right|} \exp \left(\frac{i \arg \left(k_{j, 1}-\lambda\right)}{2}\right) \\
& \times \sqrt{\left|k_{j, 2}-\lambda\right|} \exp \left(\frac{i \arg \left(\lambda-k_{j, 2}\right)}{2}\right),
\end{aligned}
$$

где функции $|\xi|$ и $\arg (\xi)$ есть модуль и аргумент комплексной переменной $\xi$.

В представлении полей (7) при любых $z$ и правильном выборе аналитической ветви (9) будет обеспечена сходимость интегралов. При этом не будут возникать нефизичные экспоненциально усиливающиеся гармоники при больших значениях $\xi$ и $\eta$.

Из уравнения $\operatorname{rot} \mathbf{E}_{j}=i \omega \mathbf{B}_{j} \quad$ и, учитывая $\hat{E}_{j, z}^{ \pm}=\mp \xi \hat{E}_{j, x}^{ \pm} / \gamma_{j} \mp \eta \hat{E}_{j, y}^{ \pm} / \gamma_{j} \quad$ (из $\left.\operatorname{div} \mathbf{E}_{j}=0\right), \quad$ найдем общее решение для магнитного поля в рассматриваемой области с номером $j$ (в пленке с номером $j-1$ ) можно записать в виде

$$
\begin{aligned}
& \mathbf{B}_{j}(x, y, z)= \\
& =\frac{1}{(2 \pi)^{2}} \int_{-\infty}^{+\infty} \int_{-\infty}^{+\infty}\left(\begin{array}{cc}
-\xi \eta / \omega \gamma_{j} & -\left(\gamma_{j}^{2}+\eta^{2}\right) / \omega \gamma_{j} \\
\left(\gamma_{j}^{2}+\xi^{2}\right) / \omega \gamma_{j} & \xi \eta / \omega \gamma_{j} \\
-\eta / \omega & \xi / \omega
\end{array}\right) \\
& \times\left(\begin{array}{c}
\hat{E}_{j, x}^{+} \\
\hat{E}_{j, y}^{+}
\end{array}\right) e^{i \gamma_{j}\left(z-z_{j-1}\right)} e^{i(\xi x+\eta y)} d \xi d \eta \\
& +\frac{1}{(2 \pi)^{2}} \int_{-\infty}^{+\infty} \int_{-\infty}^{+\infty}\left(\begin{array}{cc}
\xi \eta / \omega \gamma_{j} & \left(\gamma_{j}^{2}+\eta^{2}\right) / \omega \gamma_{j} \\
-\left(\gamma_{j}^{2}+\xi^{2}\right) / \omega \gamma_{j} & -\xi \eta / \omega \gamma_{j} \\
-\eta / \omega & \xi / \omega
\end{array}\right) \\
& \times\left(\begin{array}{c}
\hat{E}_{j, x}^{-} \\
\hat{E}_{j, y}^{-}
\end{array}\right) e^{-i \gamma_{j}\left(z-z_{j-1}\right)} e^{i(\xi x+\eta y)} d \xi d \eta .
\end{aligned}
$$

Вводя вектор-столбец $\hat{\mathfrak{E}}_{j}=\left(\hat{E}_{j, x}^{+} ; \hat{E}_{j, y}^{+} ; \hat{E}_{j, x}^{-} ; \hat{E}_{j, y}^{-}\right)^{T}$, из (7) и (10) выразим составляющие фурье-образов тангенциальных полей на границах области в следующем 
матричном виде:

$$
\begin{aligned}
&\left.\left(\begin{array}{c}
\tilde{E}_{j, x} \\
\tilde{E}_{j, y} \\
\tilde{B}_{j, x} / \mu_{j} \\
\tilde{B}_{j, y} / \mu_{j}
\end{array}\right)\right|_{z=z_{j-1}}=\left(\begin{array}{cc}
\mathbf{I} & e^{i \gamma_{j} d_{j-1} \mathbf{I}} \\
\mathbf{G}_{j} & -e^{i \gamma_{j} d_{j-1}} \mathbf{G}_{j}
\end{array}\right) \hat{\mathfrak{E}}_{j}, \\
&\left.\left(\begin{array}{c}
\tilde{E}_{j, x} \\
\tilde{E}_{j, y} \\
\tilde{B}_{j, x} / \mu_{j} \\
\tilde{B}_{j, y} / \mu_{j}
\end{array}\right)\right|_{z=z_{j}}=\left(\begin{array}{cc}
e^{i \gamma_{j} d_{j-1}} \mathbf{I} & \mathbf{I} \\
e^{i \gamma_{j} d_{j-1}} \mathbf{G}_{j} & -\mathbf{G}_{j}
\end{array}\right) \hat{\mathfrak{E}}_{j},
\end{aligned}
$$

где $d_{j-1}=z_{j}-z_{j-1}$, $\mathbf{I}-$ единичная матрица $2 \times 2$, а матрица $\mathbf{G}_{j}$ представима в виде

$$
\mathbf{G}_{j}=\left(\begin{array}{cc}
-\frac{\xi \eta}{\omega \mu_{j} \gamma_{j}} & -\frac{\gamma_{j}^{2}+\eta^{2}}{\omega \mu_{j} \gamma_{j}} \\
\frac{\gamma_{j}^{2}+\xi^{2}}{\omega \mu_{j} \gamma_{j}} & \frac{\xi \eta}{\omega \mu_{j} \gamma_{j}}
\end{array}\right) .
$$

\section{Распространение электромагнитной волны в многослойной структуре, свободной от сторонних токов}

Рассмотрим теперь многослойную структуру, внутри которой нет сторонних токов. Рассмотрим границу $z=z_{j}$ между областями с номерами $j$ и $j+1$. Уравнения непрерывности тангенциальных компонент напряженностей электрического и магнитного полей на этой границе могут быть записаны в виде:

$$
\begin{gathered}
E_{j, x}\left(x, y, z_{j}\right)-E_{j+1, x}\left(x, y, z_{j}\right)=0, \\
E_{j, y}\left(x, y, z_{j}\right)-E_{j+1, y}\left(x, y, z_{j}\right)=0, \\
B_{j, x}\left(x, y, z_{j}\right) / \mu_{j}-B_{j+1, x}\left(x, y, z_{j}\right) / \mu_{j+1}=0, \\
B_{j, y}\left(x, y, z_{j}\right) / \mu_{j}-B_{j+1, y}\left(x, y, z_{j}\right) / \mu_{j+1}=0,
\end{gathered}
$$

где электрические и магнитные поля в области $j+1$ выражаются формулами (7) и (10), в которых произведена замена индексов $j \rightarrow j+1$. Так как уравнения Максвелла - линейные уравнения, то граничные условия должны выполняться для каждого члена фурье-разложения, из которых, с помощью (11) и (12), получим матричное уравнение на границе $z=z_{j}$ :

$$
\left(\begin{array}{cc}
e^{i \gamma_{j} d_{j-1} \mathbf{I}} & \mathbf{I} \\
e^{i \gamma_{j} d_{j-1}} \mathbf{G}_{j} & -\mathbf{G}_{j}
\end{array}\right) \hat{\mathfrak{E}}_{j}=\left(\begin{array}{cc}
\mathbf{I} & e^{i \gamma_{j+1} d_{j} \mathbf{I}} \\
\mathbf{G}_{j+1} & -e^{i \gamma_{j+1} d_{j}} \mathbf{G}_{j+1}
\end{array}\right) \hat{\mathfrak{E}}_{j+1},
$$

где $d_{j-1}=z_{j}-z_{j-1}, d_{j}=z_{j+1}-z_{j}$.

Уравнение (14) можно записать для $j=2, \ldots$ $\ldots,(N-1)$, где $(N+1)$ - общее число областей, $N$ - число границ, то есть для всех границ, исключая первую $(j=1)$ и последнюю $(j=N)$ границы. То есть, исключая границу $z_{1}=0$ и $z_{N}=d_{\mathrm{tot}}=\sum_{m=1}^{N-1} d_{m}$, где $d_{\mathrm{tot}}-$ общая толщина слоистой структуры (сумма толщин пленок, составляющих рассматриваемую структуру).

Общее решение для электрического и магнитного полей в области $j=1$, то есть в интервале $\left(-\infty, z_{1}\right)$, где $z_{1}=0$, запишем в виде

$$
\begin{aligned}
\mathbf{E}_{1}(x, y, z)= & \frac{1}{(2 \pi)^{2}} \int_{-\infty}^{+\infty} \int_{-\infty}^{+\infty}\left(\begin{array}{cc}
1 & 0 \\
0 & 1 \\
-\xi / \gamma_{1} & -\eta / \gamma_{1}
\end{array}\right) \\
& \times\left(\begin{array}{c}
\hat{E}_{1, x}^{+} \\
\hat{E}_{1, y}^{+}
\end{array}\right) e^{i \gamma_{1} z} e^{i(\xi x+\eta y)} d \xi d \eta \\
& +\frac{1}{(2 \pi)^{2}} \int_{-\infty}^{+\infty} \int_{-\infty}^{+\infty}\left(\begin{array}{cc}
1 & 0 \\
0 & 1 \\
\xi / \gamma_{1} & \eta / \gamma_{1}
\end{array}\right) \\
& \times\left(\begin{array}{l}
\hat{E}_{1, x}^{-} \\
\hat{E}_{1, y}^{-}
\end{array}\right) e^{-i \gamma_{1} z} e^{i(\xi x+\eta y)} d \xi d \eta,
\end{aligned}
$$

$\mathbf{B}_{1}(x, y, z)=$

$$
=\frac{1}{(2 \pi)^{2}} \int_{-\infty}^{+\infty} \int_{-\infty}^{+\infty}\left(\begin{array}{cc}
-\xi \eta / \omega \gamma_{1} & -\left(\gamma_{1}^{2}+\eta^{2}\right) / \omega \gamma_{1} \\
\left(\gamma_{1}^{2}+\xi^{2}\right) / \omega \gamma_{1} & \xi \eta / \omega \gamma_{1} \\
-\eta / \omega & \xi / \omega
\end{array}\right)
$$$$
\times\left(\begin{array}{c}
\hat{E}_{1, x}^{+} \\
\hat{E}_{1, y}^{+}
\end{array}\right) e^{i \gamma_{1} z} e^{i(\xi x+\eta y)} d \xi d \eta
$$$$
+\frac{1}{(2 \pi)^{2}} \int_{-\infty}^{+\infty} \int_{-\infty}^{+\infty}\left(\begin{array}{cc}
\xi \eta / \omega \gamma_{1} & \left(\gamma_{1}^{2}+\eta^{2}\right) / \omega \gamma_{1} \\
-\left(\gamma_{1}^{2}+\xi^{2}\right) / \omega \gamma_{1} & -\xi \eta / \omega \gamma_{1} \\
-\eta / \omega & \xi / \omega
\end{array}\right)
$$

$$
\times\left(\begin{array}{c}
\hat{E}_{1, x}^{-} \\
\hat{E}_{1, y}^{-}
\end{array}\right) e^{-i \gamma_{1} z} e^{i(\xi x+\eta y)} d \xi d \eta
$$

Тогда граничные условия на границе $z_{1}=0$ можно записать в виде

$$
\left(\begin{array}{cc}
\mathbf{I} & \mathbf{I} \\
\mathbf{G}_{1} & -\mathbf{G}_{1}
\end{array}\right) \hat{\mathfrak{E}}_{1}=\left(\begin{array}{cc}
\mathbf{I} & e^{i \gamma_{2} d_{1}} \mathbf{I} \\
\mathbf{G}_{2} & -e^{i \gamma_{2} d_{1}} \mathbf{G}_{2}
\end{array}\right) \hat{\mathfrak{E}}_{2} .
$$


Аналогично, общее решение для электрического и магнитного полей в области $j=N+1$, то есть в интервале $\left(z_{N},+\infty\right)$, запишем в виде

$$
\begin{aligned}
& \mathbf{E}_{N+1}(x, y, z)=\frac{1}{(2 \pi)^{2}} \int_{-\infty}^{+\infty} \int_{-\infty}^{+\infty}\left(\begin{array}{cc}
1 & 0 \\
0 & 1 \\
-\xi / \gamma_{N+1} & -\eta / \gamma_{N+1}
\end{array}\right) \\
& \times\left(\begin{array}{c}
\hat{E}_{N+1, x}^{+} \\
\hat{E}_{N+1, y}^{+}
\end{array}\right) e^{i \gamma_{N+1}\left(z-z_{N}\right)} e^{i(\xi x+\eta y)} d \xi d \eta \\
& +\frac{1}{(2 \pi)^{2}} \int_{-\infty}^{+\infty} \int_{-\infty}^{+\infty}\left(\begin{array}{cc}
1 & 0 \\
0 & 1 \\
\xi / \gamma_{N+1} & \eta / \gamma_{N+1}
\end{array}\right) \\
& \times\left(\begin{array}{c}
\hat{E}_{N+1, x}^{-} \\
\hat{E}_{N+1, y}^{-}
\end{array}\right) e^{-i \gamma_{N+1}\left(z-z_{N}\right)} e^{i(\xi x+\eta y)} d \xi d \eta \\
& \mathbf{B}_{N+1}(x, y, z)= \\
& =\frac{1}{(2 \pi)^{2}} \int_{-\infty}^{+\infty} \int_{-\infty}^{+\infty}\left(\begin{array}{cc}
-\xi \eta / \omega \gamma_{N+1} & -\left(\gamma_{N+1}^{2}+\eta^{2}\right) / \omega \gamma_{N+1} \\
\left(\gamma_{N+1}^{2}+\xi^{2}\right) / \omega \gamma_{N+1} & \xi \eta / \omega \gamma_{N+1} \\
-\eta / \omega & \xi / \omega
\end{array}\right) \\
& \times\left(\begin{array}{c}
\hat{E}_{N+1, x}^{+} \\
\hat{E}_{N+1, y}^{+}
\end{array}\right) e^{i \gamma_{N+1}\left(z-z_{N}\right)} e^{i(\xi x+\eta y)} d \xi d \eta \\
& +\frac{1}{(2 \pi)^{2}} \int_{-\infty}^{+\infty} \int_{-\infty}^{+\infty}\left(\begin{array}{cc}
\xi \eta / \omega \gamma_{N+1} & \left(\gamma_{N+1}^{2}+\eta^{2}\right) / \omega \gamma_{N+1} \\
-\left(\gamma_{N+1}^{2}+\xi^{2}\right) / \omega \gamma_{N+1} & -\xi \eta / \omega \gamma_{N+1} \\
-\eta / \omega & \xi / \omega
\end{array}\right) \\
& \times\left(\begin{array}{c}
\hat{E}_{N+1, x}^{-} \\
\hat{E}_{N+1, y}^{-}
\end{array}\right) e^{-i \gamma_{N+1}\left(z-z_{N}\right)} e^{i(\xi x+\eta y)} d \xi d \eta
\end{aligned}
$$

$$
\text { (19) }
$$

Тогда, граничные условия на границе $z_{N}$ можно записать в виде

$$
\left(\begin{array}{cc}
e^{i \gamma_{N} d_{N-1} \mathbf{I}} & \mathbf{I} \\
e^{i \gamma_{N} d_{N-1}} \mathbf{G}_{N} & -\mathbf{G}_{N}
\end{array}\right) \hat{\mathfrak{E}}_{N}=\left(\begin{array}{cc}
\mathbf{I} & \mathbf{I} \\
& \\
\mathbf{G}_{N+1} & -\mathbf{G}_{N+1}
\end{array}\right) \hat{\mathfrak{E}}_{N+1} .
$$

Уравнения (14), (17) и (20) позволяют связать векторстолбцы электрического поля в первой и последней областях задачи (то есть в полупространствах, вне плоскослоистой структуры):

$$
\begin{aligned}
\hat{\mathfrak{E}}_{1} & =\left(\begin{array}{cc}
\mathbf{I} & \mathbf{I} \\
\mathbf{G}_{1} & -\mathbf{G}_{1}
\end{array}\right)^{-1} \times\left(\begin{array}{cc}
\mathbf{I} & e^{i \gamma_{2} d_{1} \mathbf{I}} \\
\mathbf{G}_{2} & -e^{i \gamma_{2} d_{1}} \mathbf{G}_{2}
\end{array}\right) \\
& \times\left(\begin{array}{cc}
e^{i \gamma_{2} d_{1} \mathbf{I}} & \mathbf{I} \\
e^{i \gamma_{2} d_{1}} \mathbf{G}_{2} & -\mathbf{G}_{2}
\end{array}\right)^{-1} \times \ldots \times\left(\begin{array}{cc}
\mathbf{I} & e^{i \gamma_{N} d_{N-1} \mathbf{I}} \\
\mathbf{G}_{N} & -e^{i \gamma_{N} d_{N-1}} \mathbf{G}_{\mathbf{N}}
\end{array}\right) \\
& \times\left(\begin{array}{cc}
e^{i \gamma_{N} d_{N-1} \mathbf{I}} & \mathbf{I} \\
e^{i \gamma_{N} d_{N-1}} \mathbf{G}_{N} & -\mathbf{G}_{N}
\end{array}\right)^{-1} \times\left(\begin{array}{cc}
\mathbf{I} & \mathbf{I} \\
\mathbf{G}_{N+1} & -\mathbf{G}_{N+1}
\end{array}\right) \hat{\mathfrak{E}}_{N+1} .
\end{aligned}
$$

или

$$
\hat{\mathfrak{E}}_{1}=\mathbf{M} \times \hat{\mathfrak{E}}_{N+1} .
$$

Матрица М имеет вид $\mathbf{M}=\mathbf{T}_{1} \times\left(\prod_{m=2}^{N} \mathbf{T}_{m}\right) \times \mathbf{T}_{N+1}$, где

$$
\begin{gathered}
\mathbf{T}_{1}=\left(\begin{array}{cc}
\mathbf{I} & \mathbf{I} \\
\mathbf{G}_{1} & -\mathbf{G}_{1}
\end{array}\right)^{-1} \\
\mathbf{T}_{m}=\left(\begin{array}{cc}
\mathbf{I} & e^{i \gamma_{m} d_{m-1} \mathbf{I}} \\
\mathbf{G}_{m} & -e^{i \gamma_{m} d_{m-1}} \mathbf{G}_{m}
\end{array}\right)\left(\begin{array}{cc}
e^{i \gamma_{m} d_{m-1} \mathbf{I}} & \mathbf{I} \\
e^{i \gamma_{m} d_{m-1}} \mathbf{G}_{m} & -\mathbf{G}_{m}
\end{array}\right)^{-1}, \\
\mathbf{T}_{N+1}=\left(\begin{array}{cc}
\mathbf{I} & \mathbf{I} \\
\mathbf{G}_{N+1} & -\mathbf{G}_{N+1}
\end{array}\right) .
\end{gathered}
$$

Если нам известна, например, падающая на плоскослоистую структуру волна, а, значит, компоненты $\hat{E}_{1, x}^{+}$ и $\hat{E}_{1, y}^{+}$вектор-столбца $\hat{\mathfrak{E}}_{1}$, то из уравнения $(21)$ можно найти компоненты $\hat{E}_{1, x}^{-}$и $\hat{E}_{1, y}^{-}$отраженной волны и саму отраженную волну по формулам (15), (16), а также компоненты $\hat{E}_{N+1, x}^{+}$и $\hat{E}_{N+1, y}^{+}$вектор-столбца $\hat{E}_{N+1}$ и прошедшую волну по формулам (18), (19). Подробности решения подобных задач, в которых рассматриваются пространственно ограниченные падающие на плоскослоистую структуру пучки можно найти, например, в работах [17-19].

\section{Распространение электромагнитной волны в плоскослоистой структуре, от элементарного источника стороннего тока, параллельного границе}

Пусть имеется точечный излучатель, расположенный в точке $\left(0,0, z_{d}\right)$ в области с номером $s$ (рис. 3$)$. Пусть этот излучатель определяется плотностью стороннего тока, параллельного границе

$$
\mathbf{J}(x, y, z)=\left(n_{x} \mathbf{e}_{x}+n_{y} \mathbf{e}_{y}\right) \delta(x) \delta(y) \delta\left(z-z_{d}\right),
$$

где $n_{x}, n_{y}$ - направляющие косинусы вектора тока вдоль осей координат $X$ и $Y, \delta(x)$ - дельта-функция Дирака. 


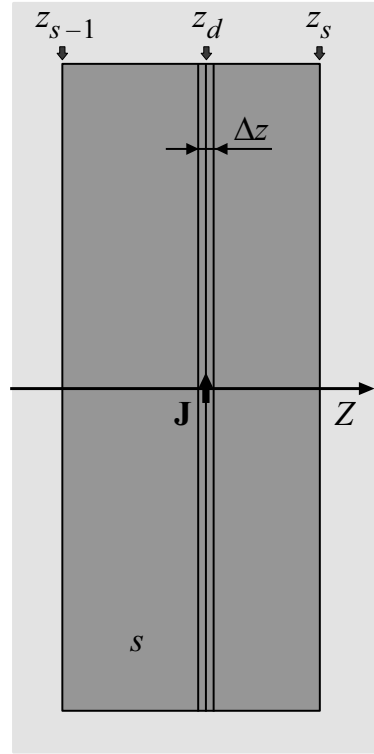

Рис. 3. Точечный излучатель, параллельный границе, расположенный в точке $\left(0,0, z_{d}\right)$ в области с номером $s$.

Тогда фурье-образы составляющих этого тока определятся следующими выражениями

$$
\begin{gathered}
\tilde{J}_{s, x}(\xi, \eta, z)=n_{x} \delta\left(z-z_{d}\right), \quad \tilde{J}_{s, y}(\xi, \eta, z)=n_{y} \delta\left(z-z_{d}\right), \\
\tilde{J}_{s, z}(\xi, \eta, z)=0 .
\end{gathered}
$$

Пусть этот элементарный источник тока находится в бесконечно тонком слое $\left(z_{d}-\Delta z / 2, z_{d}+\Delta z / 2\right)$. $\left(z_{d}-\Delta z / 2, z_{d}+\Delta z / 2\right)$. Тогда уравнения Максвелла (1) и (2) для фурье-образов полей, можно записать (при $\Delta z \rightarrow 0)$ в виде

$$
\left\{\begin{array}{l}
i \eta \tilde{E}_{s, z}-\frac{\Delta \tilde{E}_{s, y}}{\Delta z}=i \omega \tilde{B}_{s, x} \\
\frac{\Delta \tilde{E}_{s, x}}{\Delta z}-i \xi \tilde{E}_{s, z}=i \omega \tilde{B}_{s, y} \\
i \xi \tilde{E}_{s, y}-i \eta \tilde{E}_{s, x}=i \omega \tilde{B}_{s, z}
\end{array}\right.
$$

$$
\left\{\begin{array}{l}
i \eta \tilde{B}_{s, z}-\frac{\Delta B_{j, y}}{\Delta z}=\mu_{s}\left(-i \omega \varepsilon_{s} \tilde{E}_{s, x}+n_{x} \delta\left(z-z_{d}\right)\right) \\
\frac{\Delta \tilde{B}_{s, x}}{\Delta z}-i \xi \tilde{B}_{s, z}=\mu_{s}\left(-i \omega \varepsilon_{s} \tilde{E}_{s, y}+n_{y} \delta\left(z-z_{d}\right)\right) \\
i \xi \tilde{B}_{s, y}-i \eta \tilde{B}_{s, x}=\mu_{s}\left(-i \omega \varepsilon_{s} \tilde{E}_{s, z}\right) .
\end{array}\right.
$$

Так как $z$-компоненты полей из уравнений (22) и (23) можно выразить через $x$ - и $y$-компоненты по формулам

$$
\begin{gathered}
\tilde{B}_{s, z}=\frac{\xi}{\omega} \tilde{E}_{s, y}-\frac{\eta}{\omega} \tilde{E}_{s, x} \\
\tilde{E}_{s, z}=-\frac{\xi}{\omega \varepsilon_{s} \mu_{s}} \tilde{B}_{s, y}+\frac{\eta}{\omega \varepsilon_{s} \mu_{s}} \tilde{B}_{s, x}+\frac{1}{i \omega \varepsilon_{s}} \tilde{J}_{s, z},
\end{gathered}
$$

то для $x$ - и $y$-компонент напряженностей электрического и магнитного полей получим

$$
\left\{\begin{aligned}
& \Delta \tilde{E}_{s, x}=\left(\frac{i \xi \eta}{\omega \varepsilon_{s} \mu_{s}} \tilde{B}_{s, x}+i\left(\omega-\frac{\xi^{2}}{\omega \varepsilon_{s} \mu_{s}}\right) \tilde{B}_{s, y}\right) \Delta z \\
& \Delta \tilde{E}_{s, y}=\left(i\left(\frac{\eta^{2}}{\omega \varepsilon_{s} \mu_{s}}-\omega\right) \tilde{B}_{s, x}-\frac{i \xi \eta}{\omega \varepsilon_{s} \mu_{s}} \tilde{B}_{s, y}\right) \Delta z \\
& \frac{\Delta \tilde{B}_{s, x}}{\mu_{s}}=\left(-\frac{i \xi \eta}{\omega \mu_{s}} \tilde{E}_{s, x}+i\left(\frac{\xi^{2}}{\omega \mu_{s}}-\omega \varepsilon_{s}\right) \tilde{E}_{s, y}\right. \\
&\left.\quad+n_{y} \delta\left(z-z_{d}\right)\right) \Delta z \\
& \frac{\Delta \tilde{B}_{s, y}}{\mu_{s}}=\left(i\left(\omega \varepsilon_{s}-\frac{\eta^{2}}{\omega \mu_{s}}\right) \tilde{E}_{s, x}+\frac{i \xi \eta \eta}{\omega \mu_{s}} \tilde{E}_{s, y}\right. \\
&\left.-n_{x} \delta\left(z-z_{d}\right)\right) \Delta z
\end{aligned}\right.
$$

Тогда, в пределе $\Delta z \rightarrow 0$, скачок тангенциальных компонент напряженностей электрического и магнитного полей при переходе через бесконечно тонкий слой с током равен

$$
\left.\left(\begin{array}{c}
\tilde{E}_{s, x} \\
\tilde{E}_{s, y} \\
\tilde{B}_{s, x} / \mu_{s} \\
\tilde{B}_{s, y} / \mu_{s}
\end{array}\right)\right|_{z=z_{d}+0}-\left.\left(\begin{array}{c}
\tilde{E}_{s, x} \\
\tilde{E}_{s, y} \\
\tilde{B}_{s, x} / \mu_{s} \\
\tilde{B}_{s, y} / \mu_{s}
\end{array}\right)\right|_{z=z_{d}-0}=\left(\begin{array}{c}
0 \\
0 \\
n_{y} \\
-n_{x}
\end{array}\right) .
$$

Выразим теперь левую часть граничного условия (24) через вектор-столбцы $\hat{\mathfrak{E}}_{1}$ и $\hat{\mathfrak{E}}_{N+1}$ полупространств, снаружи плоскослоистой структуры. Для этого разобьем область с номером $s$ на две области и обозначим их индексами $l$ и $r$ (левая и правая, если смотреть на рис. 3). Введем вектор-столбцы $\hat{\mathfrak{E}}_{l}$ и $\hat{\mathfrak{E}}_{r}$ в этих областях. Тогда (24), учитывая (11) и (12), можно записать в виде

$$
\begin{aligned}
& \left(\begin{array}{cc}
\mathbf{I} & e^{i \gamma_{s}\left(z_{s}-z_{d}\right)} \mathbf{I} \\
\mathbf{G}_{s} & -e^{i \gamma_{s}\left(z_{s}-z_{d}\right)} \mathbf{G}_{s}
\end{array}\right) \hat{\mathfrak{E}}_{r} \\
& -\left(\begin{array}{cc}
e^{i \gamma_{s}\left(z_{d}-z_{s-1}\right)} \mathbf{I} & \mathbf{I} \\
e^{i \gamma_{s}\left(z_{d}-z_{s-1}\right)} \mathbf{G}_{s} & -\mathbf{G}_{s}
\end{array}\right) \hat{\mathfrak{E}}_{l}=\mathbf{V},
\end{aligned}
$$

где $\mathbf{V}=\left(0 ; 0 ; n_{y} ;-n_{x}\right)^{T}$. Кроме того, из (21) следует, что

$$
\hat{\mathfrak{E}}_{1}=\mathbf{Q}_{L} \hat{\mathfrak{E}}_{l},
$$

где

$$
\begin{aligned}
& \mathbf{Q}_{L}=\left(\begin{array}{cc}
\mathbf{I} & \mathbf{I} \\
\mathbf{G}_{1} & -\mathbf{G}_{1}
\end{array}\right)^{-1} \\
& \times\left(\begin{array}{cc}
\mathbf{I} & e^{i \gamma_{2} d_{1} \mathbf{I}} \\
\mathbf{G}_{2} & -e^{i \gamma_{2} d_{1}} \mathbf{G}_{2}
\end{array}\right) \times\left(\begin{array}{cc}
e^{i \gamma_{2} d_{1} \mathbf{I}} & \mathbf{I} \\
e^{i \gamma_{2} d_{1}} \mathbf{G}_{2} & -\mathbf{G}_{2}
\end{array}\right)^{-1} \\
& \times \ldots \times\left(\begin{array}{cc}
\mathbf{I} & e^{i \gamma_{s-1} d_{s-2} \mathbf{I}} \\
\mathbf{G}_{s-1} & -e^{i \gamma_{s-1} d_{s-2}} \mathbf{G}_{s-1}
\end{array}\right) \\
& \times\left(\begin{array}{cc}
e^{i \gamma_{s-1} d_{s-2} \mathbf{I}} & \mathbf{I} \\
e^{i \gamma_{s-1} d_{s-2}} \mathbf{G}_{s-1} & -\mathbf{G}_{s-1}
\end{array}\right)^{-1} \times\left(\begin{array}{cc}
\mathbf{I} & e^{i \gamma_{s}\left(z_{d}-z_{s-1}\right)} \mathbf{I} \\
\mathbf{G}_{s} & -e^{i \gamma_{s}\left(z_{d}-z_{s-1}\right)} \mathbf{G}_{s}
\end{array}\right),
\end{aligned}
$$


а также

$$
\hat{\mathfrak{E}}_{r}=\mathbf{Q}_{R} \hat{\mathfrak{E}}_{N+1},
$$

где

$$
\begin{aligned}
& \mathbf{Q}_{R}=\left(\begin{array}{cc}
e^{i \gamma_{s}\left(z_{s}-z_{d}\right)} \mathbf{I} & \mathbf{I} \\
e^{i \gamma_{s}\left(z_{s}-z_{d}\right)} \mathbf{G}_{s} & -\mathbf{G}_{s}
\end{array}\right)^{-1} \times\left(\begin{array}{cc}
\mathbf{I} & e^{i \gamma_{s+1} d_{s} \mathbf{I}} \\
\mathbf{G}_{s+1} & -e^{i \gamma_{s+1} d_{s}} \mathbf{G}_{s+1}
\end{array}\right) \\
& \times\left(\begin{array}{cc}
e^{i \gamma_{s+1} d_{s} \mathbf{I}} & \mathbf{I} \\
e^{i \gamma_{s+1} d_{s}} \mathbf{G}_{s+1} & -\mathbf{G}_{s+1}
\end{array}\right)^{-1} \times \ldots \times\left(\begin{array}{cc}
\mathbf{I} & e^{i \gamma_{N} d_{N-1} \mathbf{I}} \\
\mathbf{G}_{N} & -e^{i \gamma_{N} d_{N-1}} \mathbf{G}_{N}
\end{array}\right) \\
& \times\left(\begin{array}{cc}
e^{i \gamma_{N} d_{N-1} \mathbf{I}} & \mathbf{I} \\
e^{i \gamma_{N} d_{N-1}} \mathbf{G}_{N} & -\mathbf{G}_{N}
\end{array}\right)^{-1} \times\left(\begin{array}{cc}
\mathbf{I} & \mathbf{I} \\
\mathbf{G}_{N+1} & -\mathbf{G}_{N+1}
\end{array}\right) .
\end{aligned}
$$

Подставляя (26), (27) в (25), и затем полученные выражения в (24), получаем:

$$
\mathbf{H}_{R} \times \hat{\mathfrak{E}}_{N+1}=\mathbf{H}_{L} \times \hat{\mathfrak{E}}_{1}+\mathbf{V},
$$

где $\mathbf{V}$ - вектор-столбец, характеризующий возбуждающее воздействие на систему стороннего элементарного тока, а матрицы $\mathbf{H}_{R}$ и $\mathbf{H}_{L}-$ характеризуют отклик на внешнее возбуждение слоистой структуры справа и слева излучателя и выражаются следующим образом:

$$
\begin{gathered}
\mathbf{H}_{R}=\left(\begin{array}{cc}
\mathbf{I} & e^{i \gamma_{s}\left(z_{s}-z_{d}\right)} \mathbf{I} \\
\mathbf{G}_{s} & -e^{i \gamma_{s}\left(z_{s}-z_{d}\right)} \mathbf{G}_{s}
\end{array}\right) \mathbf{Q}_{R} \\
=\mathbf{T}_{R} \times\left(\prod_{m=s+1}^{N} \mathbf{T}_{m}\right) \times \mathbf{T}_{N+1}, \\
\mathbf{H}_{L}=\left(\begin{array}{cc}
e^{i \gamma_{s}\left(z_{d}-z_{s-1}\right)} \mathbf{I} & \mathbf{I} \\
e^{i \gamma_{s}\left(z_{d}-z_{s-1}\right)} \mathbf{G}_{s} & -\mathbf{G}_{s}
\end{array}\right)\left(\mathbf{Q}_{L}\right)^{-1} \\
=\left(\mathbf{T}_{1} \times\left(\prod_{m=2}^{s-1} \mathbf{T}_{m}\right) \times \mathbf{T}_{L}\right)^{-1},
\end{gathered}
$$

где матрицы $\mathbf{T}_{m}$ при $m \neq s$ выражаются формулой

$$
\mathbf{T}_{m}=\left(\begin{array}{cc}
\mathbf{I} & e^{i \gamma_{m} d_{m-1} \mathbf{I}} \\
\mathbf{G}_{m} & -e^{i \gamma_{m} d_{m-1}} \mathbf{G}_{m}
\end{array}\right)\left(\begin{array}{cc}
e^{i \gamma_{m} d_{m-1} \mathbf{I}} & \mathbf{I} \\
e^{i \gamma_{m} d_{m-1}} \mathbf{G}_{m} & -\mathbf{G}_{m}
\end{array}\right)^{-1},
$$

а матрицы $\mathbf{T}_{L}$ и $\mathbf{T}_{R}-$ формулами

$$
\begin{gathered}
\mathbf{T}_{L}=\left(\begin{array}{cc}
\mathbf{I} & e^{i \gamma_{s}\left(z_{d}-z_{s-1}\right)} \mathbf{I} \\
\mathbf{G}_{s} & -e^{i \gamma_{s}\left(z_{d}-z_{s-1}\right)} \mathbf{G}_{s}
\end{array}\right)\left(\begin{array}{cc}
e^{i \gamma_{s}\left(z_{d}-z_{s-1}\right)} \mathbf{I} & \mathbf{I} \\
e^{i \gamma_{s}\left(z_{d}-z_{s-1}\right)} \mathbf{G}_{s} & -\mathbf{G}_{s}
\end{array}\right)^{-1}, \\
\mathbf{T}_{R}=\left(\begin{array}{cc}
\mathbf{I} & e^{i \gamma_{s}\left(z_{s}-z_{d}\right)} \mathbf{I} \\
\mathbf{G}_{s} & -e^{i \gamma_{s}\left(z_{s}-z_{d}\right)} \mathbf{G}_{s}
\end{array}\right)\left(\begin{array}{cc}
e^{i \gamma_{s}\left(z_{s}-z_{d}\right)} \mathbf{I} & \mathbf{I} \\
e^{i \gamma_{s}\left(z_{s}-z_{d}\right)} \mathbf{G}_{s} & -\mathbf{G}_{s}
\end{array}\right)^{-1} .
\end{gathered}
$$

В вышеприведенных формулах матрицы $\mathbf{G}_{s}$ выражаются по формулам (13).

В рассматриваемой задаче источники полей находятся внутри плоскослоистой структуры. Поэтому в столбцах $\hat{\mathfrak{E}}_{1}$ и $\hat{\mathfrak{E}}_{N+1}$, есть только компоненты волн, идущие от плоскослоистой структуры. Поэтому эти столбцы имеют вид

$$
\hat{\mathfrak{E}}_{1}=\left(0 ; 0 ; \hat{E}_{1, x}^{-} ; \hat{E}_{1, y}^{-}\right)^{T}, \quad \hat{\mathfrak{E}}_{N+1}=\left(\hat{E}_{N+1, x}^{+} ; \hat{E}_{N+1, y}^{+} ; 0 ; 0\right)^{T} .
$$

Чтобы получить оставшиеся отличные от нуля компоненты $\hat{\mathfrak{E}}_{1}$ и $\hat{\mathfrak{E}}_{N+1}$ разобьем матрицы $\mathbf{H}_{R}$ и $\mathbf{H}_{L}$ каждую на четыре подматрицы $2 \times 2$ следующим образом:

$$
\mathbf{H}_{R}=\left(\begin{array}{ll}
\mathbf{H}_{R A} & \mathbf{H}_{R B} \\
\mathbf{H}_{R C} & \mathbf{H}_{R D}
\end{array}\right), \quad \mathbf{H}_{L}=\left(\begin{array}{cc}
\mathbf{H}_{L A} & \mathbf{H}_{L B} \\
\mathbf{H}_{L C} & \mathbf{H}_{L D}
\end{array}\right) .
$$

тогда уравнение (28) примет вид

$$
\left(\begin{array}{ll}
\mathbf{H}_{R A} & \mathbf{H}_{R B} \\
\mathbf{H}_{R C} & \mathbf{H}_{R D}
\end{array}\right) \times\left(\begin{array}{c}
\hat{E}_{N+1, x}^{+} \\
\hat{E}_{N+1, y}^{+} \\
0 \\
0
\end{array}\right)=\left(\begin{array}{cc}
\mathbf{H}_{L A} & \mathbf{H}_{L B} \\
\mathbf{H}_{L C} & \mathbf{H}_{L D}
\end{array}\right) \times\left(\begin{array}{c}
0 \\
0 \\
\hat{E}_{1, x}^{-} \\
\hat{E}_{1, y}^{-}
\end{array}\right)+\mathbf{V} .
$$

Если еще разбить вектор $\mathbf{V}=\left(V_{1} ; V_{2} ; V_{3} ; V_{4}\right)^{T}$ на $\mathbf{V}_{A}=\left(V_{1}, V_{2}\right)^{T}$ и $\mathbf{V}_{B}=\left(V_{3}, V_{4}\right)^{T}$, то уравнение (29) можно представить следующей системой из двух матричных уравнений:

$$
\begin{aligned}
& \mathbf{H}_{R A}\left(\begin{array}{c}
\hat{E}_{N+1, x}^{+} \\
\hat{E}_{N+1, y}^{+}
\end{array}\right)=\mathbf{H}_{L B}\left(\begin{array}{c}
\hat{E}_{1, x}^{-} \\
\hat{E}_{1, y}^{-}
\end{array}\right)+\mathbf{V}_{A}, \\
& \mathbf{H}_{R C}\left(\begin{array}{c}
\hat{E}_{N+1, x}^{+} \\
\hat{E}_{N+1, y}^{+}
\end{array}\right)=\mathbf{H}_{L D}\left(\begin{array}{c}
\hat{E}_{1, x}^{-} \\
\hat{E}_{1, y}^{-}
\end{array}\right)+\mathbf{V}_{B} .
\end{aligned}
$$

Полученные уравнения можно снова объединить в одно матричное уравнение $4 \times 4$ :

$$
\left(\begin{array}{cc}
-\mathbf{H}_{L B} & \mathbf{H}_{R A} \\
-\mathbf{H}_{L D} & \mathbf{H}_{R C}
\end{array}\right) \hat{\mathfrak{E}}_{\text {out }}=\mathbf{V}
$$

$\stackrel{\text { где }}{\hat{\mathfrak{E}}_{\text {out }}}=\left(\hat{E}_{1, x}^{-} ; \hat{E}_{1, y}^{-} ; \hat{E}_{N+1, x}^{+} ; \hat{E}_{N+1, y}^{+}\right)^{T}$.

Решая это уравнение, найдем $\hat{E}_{1, x}^{-}, \hat{E}_{1, y}^{-}$и $\hat{E}_{N+1, x}^{+}$, $\hat{E}_{N+1, y}^{+}$, а значит, волну, уходящую из плоскослоистой структуры влево (в направлении $z \rightarrow-\infty$ ), по формулам

$$
\begin{aligned}
\mathbf{E}_{1}(x, y, z) & =\frac{1}{(2 \pi)^{2}} \int_{-\infty}^{+\infty} \int_{-\infty}^{+\infty}\left(\begin{array}{cc}
1 & 0 \\
0 & 1 \\
\xi / \gamma_{1} & \eta / \gamma_{1}
\end{array}\right) \\
& \times\left(\begin{array}{c}
\hat{E}_{1, x}^{-} \\
\hat{E}_{1, y}^{-}
\end{array}\right) e^{-i \gamma_{1} z} e^{i(\xi x+\eta y)} d \xi d \eta
\end{aligned}
$$




$$
\begin{aligned}
& \mathbf{B}_{1}(x, y, z)= \\
& =\frac{1}{(2 \pi)^{2}} \int_{-\infty}^{+\infty} \int_{-\infty}^{+\infty}\left(\begin{array}{cc}
\xi \eta / \omega \gamma_{1} & \left(\gamma_{1}^{2}+\eta^{2}\right) / \omega \gamma_{1} \\
-\left(\gamma_{1}^{2}+\xi^{2}\right) / \omega \gamma_{1} & -\xi \eta / \omega \gamma_{1} \\
-\eta / \omega & \xi / \omega
\end{array}\right) \\
& \quad \times\left(\begin{array}{l}
\hat{E}_{1, x}^{-} \\
\hat{E}_{1, y}^{-}
\end{array}\right) e^{-i \gamma_{1} z} e^{i(\xi x+\eta y)} d \xi d \eta
\end{aligned}
$$

и волну вправо от плоскослоистой структуры (в направлении $z \rightarrow+\infty$ ) по формулам

$$
\begin{aligned}
\mathbf{E}_{N+1}(x, y, z) & =\frac{1}{(2 \pi)^{2}} \int_{-\infty}^{+\infty} \int_{-\infty}^{+\infty}\left(\begin{array}{cc}
1 & 0 \\
0 & 1 \\
-\xi / \gamma_{N+1} & -\eta / \gamma_{N+1}
\end{array}\right) \\
& \times\left(\begin{array}{c}
\hat{E}_{N+1, x}^{+} \\
\hat{E}_{N+1, y}^{+}
\end{array}\right) e^{i \gamma_{N+1}\left(z-z_{N}\right)} e^{i(\xi x+\eta y)} d \xi d \eta,
\end{aligned}
$$

$\mathbf{B}_{N+1}(x, y, z)=$

$=\frac{1}{(2 \pi)^{2}} \int_{-\infty-\infty}^{+\infty+\infty}\left(\begin{array}{cc}-\xi \eta / \omega \gamma_{N+1} & -\left(\gamma_{N+1}^{2}+\eta^{2}\right) / \omega \gamma_{N+1} \\ \left(\gamma_{N+1}^{2}+\xi^{2}\right) / \omega \gamma_{N+1} & \xi \eta / \omega \gamma_{N+1} \\ -\eta / \omega & \xi / \omega\end{array}\right)$

$\times\left(\begin{array}{c}\hat{E}_{N+1, x}^{+} \\ \hat{E}_{N+1, y}^{+}\end{array}\right) e^{i \gamma_{N+1}\left(z-z_{N}\right)} e^{i(\xi x+\eta y)} d \xi d \eta$

Наконец, при необходимости, зная $\hat{\mathfrak{E}}_{1}$ и $\hat{\mathfrak{E}}_{N+1}$, можно найти вектор-столбцы поля в любой внутренней области $\hat{\mathfrak{E}}_{j}$, так как они однозначно определяется граничными условиями. После этого, электромагнитные поля в любой из этих областей могут быть найдены по формулам (7) и (10). Таким образом, электромагнитные поля будут определены во всем пространстве.

Отметим, что в частном случае, когда элементарный источник находится на левой свободной границе плоскослоистой структуры. В этом случае в уравнениях (28) и (30) матрицы $\mathbf{H}_{R}$ и $\mathbf{H}_{L}$ будут выражаться в виде:

$$
\mathbf{H}_{R}=\left(\prod_{m=2}^{N} \mathbf{T}_{m}\right) \times \mathbf{T}_{N+1}, \quad \mathbf{H}_{L}=\left(\mathbf{T}_{1}\right)^{-1} .
$$

\section{Поля в дальней зоне в полупространствах, окружающих плоскослоистую структуру}

Заметим, что из формул (31), (33) мы можем найти компоненты тангенциального электрического поля на границах полупространств по формулам: на границе

$$
\begin{aligned}
& z=z_{0}=0 \\
& \left\{\begin{array}{l}
E_{1, x}(x, y, 0)=\frac{1}{(2 \pi)^{2}} \int_{-\infty}^{+\infty} \int_{-\infty}^{+\infty} \hat{E}_{1, x}^{-} e^{i(\xi x+\eta y)} d \xi d \eta, \\
E_{1, y}(x, y, 0)=\frac{1}{(2 \pi)^{2}} \int_{-\infty}^{+\infty} \int_{-\infty}^{+\infty} \hat{E}_{1, y}^{-} e^{i(\xi x+\eta y)} d \xi d \eta,
\end{array}\right.
\end{aligned}
$$

на границе $z=z_{N}=d_{\text {tot }}$

$$
\left\{\begin{array}{l}
E_{N+1, x}\left(x, y, z_{N}\right)=\frac{1}{(2 \pi)^{2}} \int_{-\infty}^{+\infty} \int_{-\infty}^{+\infty} \hat{E}_{N+1, x}^{+} e^{i(\xi x+\eta y)} d \xi d \eta, \\
E_{N+1, y}\left(x, y, z_{N}\right)=\frac{1}{(2 \pi)^{2}} \int_{-\infty-\infty}^{+\infty+\infty} \int_{-\infty}^{+\infty} \hat{E}_{N+1, y}^{+} e^{i(\xi x+\eta y)} d \xi d \eta .
\end{array}\right.
$$

Тогда в полупространствах мы можем воспользоваться принципом эквивалентности и воспользоваться формулами Стрэттона и Чу [20,21] в формулировке для полуплоскости [22,23], согласно которой электромагнитные поля в полупространствах могут быть найдены только по распределению тангенциального электрического поля на границе по формулам:

$$
\begin{gathered}
\mathbf{E}(\mathbf{r})=-\operatorname{rot} \mathbf{A}_{m}(\mathbf{r}), \\
\mathbf{H}(\mathbf{r})=i \omega \varepsilon_{j} \mathbf{A}_{m}(\mathbf{r})-\frac{1}{i \omega \mu_{j}} \operatorname{grad} \operatorname{div} \mathbf{A}_{m}(\mathbf{r}) .
\end{gathered}
$$

где

$$
\mathbf{A}_{m}(\mathbf{r})=\frac{1}{2 \pi} \int_{P}[\mathbf{E}, \mathbf{n}] \frac{e^{i k_{j}\left|\mathbf{r}-\mathbf{r}_{p}\right|}}{\left|\mathbf{r}-\mathbf{r}_{p}\right|} d s_{p}
$$

n - внутренняя нормаль рассматриваемого полупространства, $\quad \mathbf{r}=x \mathbf{e}_{x}+y \mathbf{e}_{y}+z \mathbf{e}_{z}$ радиус-вектор точки наблюдения, в которой находятся поля, $\quad \mathbf{r}_{p}=x_{p} \mathbf{e}_{x}+y_{p} \mathbf{e}_{y}+z_{p} \mathbf{e}$ радиус-вектор точки интегрирования, $\left|\mathbf{r}-\mathbf{r}_{p}\right|=\sqrt{\left(x-x_{p}\right)^{2}+\left(y-y_{p}\right)^{2}+\left(z-z_{p}\right)^{2}}$ расстояние между точками $\mathbf{r}$ и $\mathbf{r}_{p}$, причем дифференцирование в операторах производится по координатам точки наблюдения, а интегрирование идет по всей плоской границе $P$ пленки.

Замечая, что $[\mathbf{E}, \mathbf{n}]=\mathbf{E}_{\tau}-$ тангенциальная составляющая поля на границе, в полупространстве $N+1$ получим

$$
\left[\mathbf{E}_{\tau}\right]=\left|\begin{array}{ccc}
\mathbf{e}_{x} & \mathbf{e}_{y} & \mathbf{e}_{z} \\
E_{N+1, x} & E_{N+1, y} & 0 \\
0 & 0 & 1
\end{array}\right|=E_{N+1, y} \mathbf{e}_{x}-E_{N+1, x} \mathbf{e}_{y},
$$




$$
\begin{aligned}
& \mathbf{A}_{m}(\mathbf{r})=\mathbf{e}_{x}\left(\frac{1}{2 \pi} \int_{-\infty-\infty}^{+\infty+\infty} \int_{-\infty+1, y}\left(x_{p}, y_{p}, z_{N}\right)\right. \\
& \left.\times \frac{e^{i k_{N+1} \sqrt{\left(x-x_{p}\right)^{2}+\left(y-y_{p}\right)^{2}+(z-d)^{2}}}}{\sqrt{\left(x-x_{p}\right)^{2}+\left(y-y_{p}\right)^{2}+\left(z-z_{N}\right)^{2}}} d x_{p} d y_{p}\right) \\
& -\mathbf{e}_{y}\left(\frac{1}{2 \pi} \int_{-\infty-\infty}^{+\infty+\infty} \int_{N+1, x}\left(x_{p}, y_{p}, z_{N}\right)\right. \\
& \left.\times \frac{e^{i k_{N+1} \sqrt{\left(x-x_{p}\right)^{2}+\left(y-y_{p}\right)^{2}+(z-d)^{2}}}}{\sqrt{\left(x-x_{p}\right)^{2}+\left(y-y_{p}\right)^{2}+\left(z-z_{N}\right)^{2}}} d x_{p} d y_{p}\right),
\end{aligned}
$$

или

$$
\begin{aligned}
& \mathbf{A}_{m}(\mathbf{r})=\mathbf{e}_{x}\left(\frac{1}{2 \pi} \int_{-\infty}^{+\infty} \int_{-\infty}^{+\infty} E_{N+1, y}\left(x_{p}, y_{p}, z_{N}\right) \frac{e^{i k_{N+1}\left|\mathbf{r}-\mathbf{r}_{p}\right|}}{\mathbf{r}-\mathbf{r}_{p}} d x_{p} d y_{p}\right) \\
&-\mathbf{e}_{y}\left(\frac{1}{2 \pi} \int_{-\infty}^{+\infty} \int_{-\infty}^{\infty} E_{N+1, x}\left(x_{p}, y_{p}, z_{N}\right) \frac{e^{i k_{N+1}\left|\mathbf{r}-\mathbf{r}_{p}\right|}}{\mathbf{r}-\mathbf{r}_{p}} d x_{p} d y_{p}\right)
\end{aligned}
$$

Тогда, по формуле $\mathbf{E}(\mathbf{r})=-\operatorname{rot} \mathbf{A}_{m}(\mathbf{r})$, составляющие электрического поля определятся следующими выражениями

$$
\begin{aligned}
& E_{N+1, x}(x, y, z)=\frac{1}{2 \pi} \int_{-\infty-\infty}^{+\infty} \int_{-\infty}^{+\infty} E_{N+1, x}\left(x_{p}, y_{p}, z_{N}\right) \\
& \times \frac{\left(z-z_{p}\right)\left(1-i k_{N+1}\left|\mathbf{r}-\mathbf{r}_{p}\right|\right)}{\left|\mathbf{r}-\mathbf{r}_{p}\right|^{3}} e^{i k_{N+1}\left|\mathbf{r}-\mathbf{r}_{p}\right|} d x_{p} d y_{p}, \quad(38) \\
& E_{N+1, y}(x, y, z)=\frac{1}{2 \pi} \int_{-\infty-\infty}^{+\infty} \int_{N+1, y}\left(x_{p}, y_{p}, z_{N}\right) \\
& \times \frac{\left(z-z_{p}\right)\left(1-i k_{N+1}\left|\mathbf{r}-\mathbf{r}_{p}\right|\right)}{\left|\mathbf{r}-\mathbf{r}_{p}\right|^{3}} e^{i k_{N+1}\left|\mathbf{r}-\mathbf{r}_{p}\right|} d x_{p} d y_{p}, \quad(39) \\
& E_{N+1, z}(x, y, z)=-\frac{1}{2 \pi} \int_{-\infty}^{+\infty} \int_{-\infty}^{+\infty}\left(\left(x-x_{p}\right) E_{N+1, x}\left(x_{p}, y_{p}, z_{N}\right)\right. \\
& \left.+\left(y-y_{p}\right) E_{N+1, y}\left(x_{p}, y_{p}, z_{N}\right)\right) \\
& \times \frac{\left(1-i k_{N+1}\left|\mathbf{r}-\mathbf{r}_{p}\right|\right) e^{i k_{N+1}\left|\mathbf{r}-\mathbf{r}_{p}\right|}}{\left|\mathbf{r}-\mathbf{r}_{p}\right|^{3}} d x_{p} d y_{p} .
\end{aligned}
$$

В принципе, выражения (38)-(40) позволяют точно определить электрическое поле в любой точке полупространства, если подставить в них выражения (37), однако, с вычислительной точки зрения, четырехкратное интегрирование не является привлекательным. Значительное упрощение получается при рассмотрении полей в дальней зоне полученной излучающей плоской апертуры. Действительно, рассмотрим поля в дальней зоне

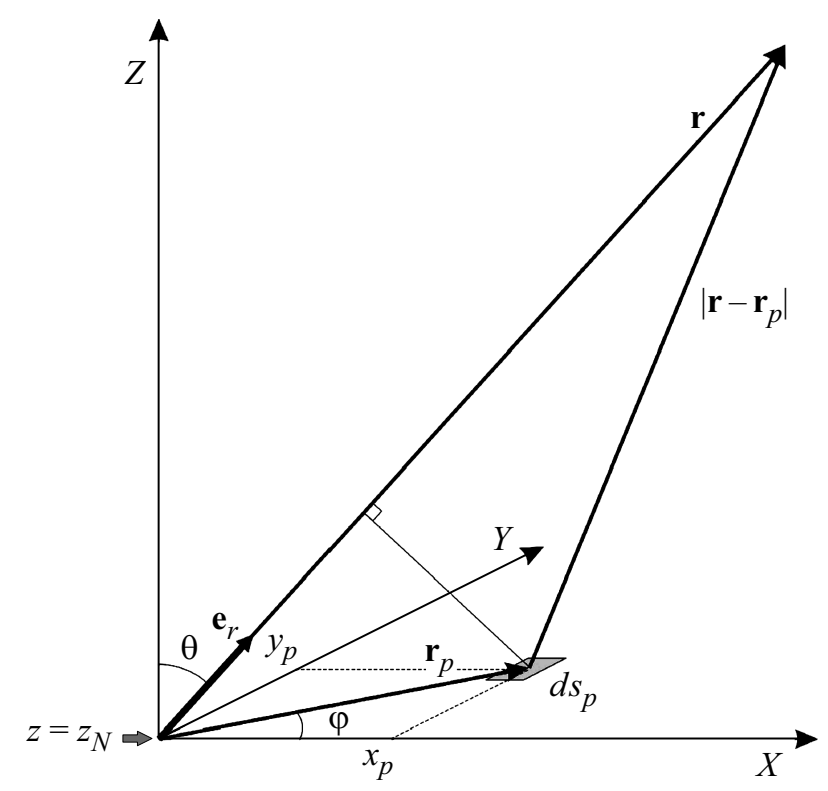

Рис. 4. Геометрия задачи нахождения электрического поля излучения в дальней зоне при интегрировании.

источника $|\mathbf{r}| \gg\left|\mathbf{r}_{p}\right|$, причем $\mathbf{r}=x \mathbf{e}_{x}+y \mathbf{e}_{y}+\left(z-z_{N}\right) \mathbf{e}_{z}$ (рис. 4) в полупространстве $N+1$.

Будем считать, что тангенциальное электрическое поле сосредоточено в основном в вблизи источника. Тогда, в подынтегральном выражении в (38) получим в пределе дальней зоны, где $\mathbf{r}$ и $\mathbf{r}_{p}$ практически параллельны, выражения

$$
\begin{aligned}
& \frac{\left(z-z_{N}\right)\left(1-i k_{N+1}\left|\mathbf{r}-\mathbf{r}_{p}\right|\right)}{\left|\mathbf{r}-\mathbf{r}_{p}\right|^{3}} e^{i k_{N+1}\left|\mathbf{r}-\mathbf{r}_{p}\right|} \rightarrow \\
& \rightarrow-i k_{N+1} \frac{\left(z-z_{N}\right)}{|\mathbf{r}|} \frac{e^{i k_{N+1}|\mathbf{r}|}}{|\mathbf{r}|} e^{i\left(\mathbf{k}_{N+1} \mathbf{r}_{p}\right)} \\
& =i k_{N+1} \cos \theta \frac{e^{i k_{N+1}|\mathbf{r}|}}{|\mathbf{r}|} e^{i\left(\mathbf{k}_{N+1} \mathbf{r}_{p}\right)},
\end{aligned}
$$

где $\theta-$ полярный угол, а вектор $\mathbf{k}_{N+1}$ равен по модулю $k_{N+1}=\omega \sqrt{\varepsilon_{N+1} \mu_{N+1}}$ и направлен вдоль единичного вектора $\mathbf{e}_{r}=\mathbf{r} /|\mathbf{r}|$. Тогда получим в дальней зоне

$$
\begin{aligned}
& E_{N+1, x}(x, y, z) \approx-\frac{i k_{N+1} \cos \theta e^{i k_{N+1}|\mathbf{r}|}}{2 \pi|\mathbf{r}|} \\
& \quad \times \int_{-\infty}^{+\infty} \int_{-\infty}^{+\infty} E_{N+1, x}\left(x_{p}, y_{p}, z_{N}\right) e^{-i\left(\mathbf{k}_{N+1}, \mathbf{r}_{p}\right)} d x_{p} d y_{p} .
\end{aligned}
$$


Рассмотрим подробнее двойной интеграл в (41):

$$
\begin{aligned}
& \int_{-\infty}^{+\infty} \int_{-\infty}^{+\infty} E_{N+1, x}\left(x_{p}, y_{p}, z_{N}\right) e^{-i\left(\mathbf{k}_{N+1}, \mathbf{r}_{p}\right)} d x_{p} d y_{p} \\
& =\int_{-\infty}^{+\infty} \int_{-\infty}^{+\infty} E_{N+1, x}\left(x_{p}, y_{p}, z_{N}\right) \\
& \times e^{-i\left(x_{p} k_{N+1} \sin \theta \cos \varphi+y_{p} k_{N+1} \sin \theta \sin \varphi\right)} d x_{p} d y_{p},
\end{aligned}
$$

где $\varphi-$ угол в плоскости $X Y$ сферической систему координат (рис. 4).

Подставляя выражение (37), получим

$$
\begin{aligned}
& \int_{-\infty}^{+\infty} \int_{-\infty}^{+\infty} E_{N+1, x}\left(x_{p}, y_{p}, z_{N}\right) e^{-i\left(\mathbf{k}_{N+1}, \mathbf{r}_{p}\right)} d x_{p} d y_{p} \\
& =\frac{1}{(2 \pi)^{2}} \int_{-\infty-\infty}^{+\infty} \int_{-\infty}^{+\infty}\left(\int_{-\infty}^{+\infty} \int_{-\infty}^{+\infty} \hat{E}_{N+1, x}^{+}(\xi, \eta) e^{i\left(\xi x_{p}+\eta y_{p}\right)} d \xi d \eta\right) \\
& \times e^{-i\left(x_{p} k_{N+1} \sin \theta \cos \varphi+y_{p} k_{N+1} \sin \theta \sin \varphi\right)} d x_{p} d y_{p} \\
& =\frac{1}{(2 \pi)^{2}} \int_{-\infty-\infty}^{+\infty+\infty} \int_{N+1, x}^{+}(\xi, \eta) \\
& \times\left(\int_{-\infty}^{+\infty} \int^{i\left(\left(\xi-k_{N+1} \sin \theta \cos \varphi\right) x_{p}+\left(\eta-k_{N+1} \sin \theta \sin \varphi\right) y_{p}\right)} d x_{p} d y_{p}\right) d \xi d \eta .
\end{aligned}
$$

Учитывая известное свойство $\delta$-функции

$$
\begin{aligned}
& \left.\int_{-\infty}^{+\infty} \int_{-\infty}^{+\infty} e^{i\left(\left(\xi-k_{N+1} \sin \theta \cos \varphi\right) x_{p}+\left(\eta-k_{N+1} \sin \theta \sin \varphi\right) y_{p}\right)} d x_{p} d y_{p}\right) d \xi d \eta \\
& =(2 \pi)^{2} \delta\left(\xi-k_{N+1} \sin \theta \cos \varphi\right) \delta\left(\eta-k_{N+1} \sin \theta \sin \varphi\right)
\end{aligned}
$$

получим

$$
\begin{aligned}
& \int_{-\infty-\infty}^{+\infty} \int_{-\infty}^{+\infty} E_{N+1, x}\left(x_{p}, y_{p}, z_{N}\right) e^{-i\left(\mathbf{k}_{N+1}, \mathbf{r}_{p}\right)} d x_{p} d y_{p} \\
& \quad=\hat{E}_{N+1, x}^{+}\left(k_{N+1} \sin \theta \cos \varphi, k_{N+1} \sin \theta \sin \varphi\right),
\end{aligned}
$$

где $\hat{E}_{N+1, x}^{+}(\xi, \eta)$ - известная из решения уравнения (30) функция.

Тогда для элементарного диполя составляющая $E_{N+1, x}$ электрического поля в рассматриваемом полупространстве в дальней зоне вычисляется по формуле

$$
\begin{aligned}
& E_{N+1, x}(x, y, z) \approx-\frac{i k_{N+1} \cos \theta e^{i k_{N+1}|\mathbf{r}|}}{2 \pi|\mathbf{r}|} \\
& \times \hat{E}_{N+1, x}^{+}\left(k_{N+1} \sin \theta \cos \varphi, k_{N+1} \sin \theta \sin \varphi\right) .
\end{aligned}
$$

Аналогично для составляющей $E_{N+1, y}$ в дальней зоне получим формулу

$$
\begin{aligned}
& E_{N+1, y}(x, y, z) \approx-\frac{i k_{N+1} \cos \theta e^{i k_{N+1}|\mathbf{r}|}}{2 \pi|\mathbf{r}|} \\
& \times \hat{E}_{N+1, y}^{+}\left(k_{N+1} \sin \theta \cos \varphi, k_{N+1} \sin \theta \sin \varphi\right) .
\end{aligned}
$$

Наконец, для составляющей $E_{N+1, z}$ в дальней зоне получим формулу

$$
\begin{aligned}
& E_{N+1, z}(x, y, z) \approx \frac{i k_{N+1} \sin \theta e^{i k_{N+1}|\mathbf{r}|}}{2 \pi|\mathbf{r}|} \\
& \times\left(\cos \varphi \hat{E}_{N+1, x}^{+}\left(k_{N+1} \sin \theta \cos \varphi, k_{N+1} \sin \theta \sin \varphi\right)\right. \\
& \left.+\sin \varphi \hat{E}_{N+1}^{+}\left(k_{N+1} \sin \theta \cos \varphi, k_{N+1} \sin \theta \sin \varphi\right)\right) .
\end{aligned}
$$

Итак, мы нашли составляющие электрического поля в точке с координатами $x=r \sin \theta \cos \varphi, y=r \sin \theta \sin \varphi$, $z=r \cos \theta+z_{N}$.

Действуя аналогично, учитывая, что для полупространства $j=1$ внутренняя нормаль изменит знак, найдем электрическое поле в дальней зоне по следующим формулам

$$
\begin{aligned}
& E_{1, x}(x, y, z) \approx \frac{i k_{1} \cos \theta e^{i k_{1}|\mathbf{r}|}}{2 \pi|\mathbf{r}|} \\
& \quad \times \hat{E}_{1, x}^{-}\left(k_{1} \sin \theta \cos \varphi, k_{1} \sin \theta \sin \varphi\right),
\end{aligned}
$$

$$
\begin{aligned}
& E_{1, y}(x, y, z) \approx \frac{i k_{1} \cos \theta e^{i k_{1}|\mathbf{r}|}}{2 \pi|\mathbf{r}|} \\
& \times \hat{E}_{1, y}^{-}\left(k_{1} \sin \theta \cos \varphi, k_{1} \sin \theta \sin \varphi\right),
\end{aligned}
$$

$$
\begin{aligned}
& E_{1, z}(x, y, z) \approx-\frac{i k_{1} \sin \theta e^{i k_{1}|\mathbf{r}|}}{2 \pi|\mathbf{r}|} \\
& \times\left(\cos \varphi \hat{E}_{1, x}^{-}\left(k_{1} \sin \theta \cos \varphi, k_{1} \sin \theta \sin \varphi\right)\right. \\
& \left.+\sin \varphi \hat{E}_{1, y}^{-}\left(k_{1} \sin \theta \cos \varphi, k_{1} \sin \theta \sin \varphi\right)\right),
\end{aligned}
$$

где координаты точки, в которой находится поле вычисляют по формулам $x=r \sin \theta \cos \varphi, y=r \sin \theta \sin \varphi$, $z=r \cos \theta$, причем $\pi / 2 \leq \theta \leq \pi$ в области $j=1$, а функции $\hat{E}_{1, x}^{-}(\xi, \eta)$ и $\hat{E}_{1, y}^{-}(\xi, \eta)$ решения уравнения $(30)$.

Итак, мы получили выражения для электрических полей, излученные элементарным диполем в плоскослоистой структуре, в окружающие полупространства в дальней зоне диполя. Отметим, что полученные выражения не требуют интегрирования и требуют решения только матричного уравнения (30). 


\section{Излучение произвольного распределения сторонних токов в дальней зоне, в направлении по нормали к поверхности плоскослоистой структуры}

Пусть нам известны распределения $\hat{E}_{1, x}^{-}(\xi, \eta), \quad \hat{E}_{1, y}^{-}(\xi, \eta), \quad \hat{E}_{N+1, x}^{+}(\xi, \eta) \quad$ и $\quad \hat{E}_{N+1, y}^{+}(\xi, \eta)$ от точечного единичного источника тока $\mathbf{J}_{\delta, x}(x, y, z)=\mathbf{e}_{x} \delta(x) \delta(y) \delta\left(z-z_{N}\right) \quad$ на поверхности $z=z_{N}$. Обозначим эти распределения как $\hat{\epsilon}_{1, x}(\xi, \eta)$, $\hat{\epsilon}_{1, y}^{-}(\xi, \eta), \hat{\epsilon}_{N+1}^{+}(\xi, \eta)$ и $\hat{\epsilon}_{N+1, y}^{+}(\xi, \eta)$, соответственно. Пусть также нам известны распределения $\hat{E}_{1, x}^{-}(\xi, \eta), \hat{E}_{1, y}^{-}(\xi, \eta)$, $\hat{E}_{N+1, x}^{+}(\xi, \eta)$ и $\hat{E}_{N+1, y}^{+}(\xi, \eta)$ от точечного единичного источника тока $\mathbf{J}_{\delta, y}(x, y, z)=\mathbf{e}_{y} \delta(x) \delta(y) \delta\left(z-z_{N}\right)$ на той же поверхности $z=z_{N}$. Обозначим эти распределения как $\mathscr{\mathscr { E }}_{1, x}^{-}(\xi, \eta), \quad \hat{\mathscr{E}}_{1, y}^{-}(\xi, \eta), \quad \hat{\mathscr{E}}_{N+1, x}^{+}(\xi, \eta)$ и $\hat{\mathscr{E}}_{N+1, y}^{+}(\xi, \eta)$, соответственно. Тогда, поля в дальней зоне в направлении нормали (при этом $|\mathbf{r} \rightarrow \infty|$, $\theta \rightarrow 0)$ от распределения токов по поверхности $S$ на поверхности $z=z_{N}$ запишется по формулам (42)-(47) в виде

$$
\begin{aligned}
& E_{N+1, x}(x, y, z) \underset{|\mathbf{r}| \rightarrow \infty}{\longrightarrow}-\frac{i k_{N+1} e^{i k_{N+1}|\mathbf{r}|}}{2 \pi|\mathbf{r}|} \\
& \times \int_{S}\left\{J_{x} \hat{\epsilon}_{N+1, x}^{+}(0,0)+J_{y} \hat{\mathscr{E}}_{N+1, x}^{+}(0,0)\right\} d x \\
& E_{N+1, y}(x, y, z) \underset{|\mathbf{r}| \rightarrow \infty}{\longrightarrow}-\frac{i k_{N+1} e^{i k_{N+1}|\mathbf{r}|}}{2 \pi|\mathbf{r}|} \\
& \times \int_{S}\left\{J_{x} \hat{\epsilon}_{N+1, y}^{+}(0,0)+J_{y} \hat{\mathscr{E}}_{N+1, y}^{+}(0,0)\right\} d s, \\
& E_{N+1, z}(x, y, z) \underset{|\mathbf{r}| \rightarrow \infty}{\longrightarrow} 0, \\
& E_{1, x}(x, y, z) \underset{|\mathbf{r}| \rightarrow \infty}{\longrightarrow} \frac{i k_{1} e^{i k_{1}|\mathbf{r}|}}{2 \pi|\mathbf{r}|} \\
& \times \int_{S}\left\{J_{x} \hat{\epsilon}_{1, x}^{-}(0,0)+J_{y} \hat{\mathscr{E}}_{1, x}^{+}(0,0)\right\} d s, \\
& E_{1, y}(x, y, z) \underset{|\mathbf{r}| \rightarrow \infty}{\longrightarrow} \frac{i k_{1} e^{i k_{1}|\mathbf{r}|}}{2 \pi|\mathbf{r}|} \\
& \times \int_{S}\left\{J_{x} \hat{\epsilon}_{1, y}^{-}(0,0)+J_{y} \hat{\mathscr{E}}_{1, y}^{+}(0,0)\right\} d s, \\
& E_{1, z}(x, y, z) \underset{|\mathbf{r}| \rightarrow \infty}{\longrightarrow} 0 .
\end{aligned}
$$

Отметим важное соотношение, верное для непоглощающих сред. Найдем отношение модулей полей на одном и том же большом расстоянии $R$ по нормали от излучателя:

$$
\begin{aligned}
& \frac{\left|E_{N+1, x}\right|}{\left|E_{1, x}\right|} \underset{R \rightarrow \infty}{\longrightarrow} \frac{\left|k_{N+1}\right|}{\left|k_{1}\right|} \frac{\left|\int_{S}\left\{J_{x} \hat{\epsilon}_{N+1, x}^{+}(0,0)+J_{y} \hat{\mathscr{E}}_{N+1, x}^{+}(0,0)\right\} d s\right|}{\left|\int_{S}\left\{J_{x} \hat{\epsilon}_{1, x}^{-}(0,0)+J_{y} \hat{\mathscr{E}}_{1, x}^{-}(0,0)\right\} d s\right|} \\
& =\frac{\left|k_{N+1}\right|}{\left|k_{1}\right|} \frac{\left|\hat{\epsilon}_{N+1, x}^{+}(0,0)\right|}{\left|\hat{\epsilon}_{1, x}^{-}(0,0)\right|}, \\
& \frac{\left|E_{N+1, y}\right|}{\mid E_{1, y}} \underset{R \rightarrow \infty}{\longrightarrow} \frac{\left|k_{N+1}\right|}{\left|k_{1}\right|} \frac{\left|\int_{S}\left\{J_{x} \hat{\epsilon}_{N+1, y}^{+}(0,0)+J_{y} \hat{\mathscr{E}}_{N+1, y}^{+}(0,0)\right\} d s\right|}{\left|\int_{S}\left\{J_{x} \hat{\epsilon}_{1, y}^{-}(0,0)+J_{y} \hat{\mathscr{E}}_{1, y}^{-}(0,0)\right\} d s\right|} \\
& =\frac{\left|k_{N+1}\right|}{\left|k_{1}\right|} \frac{\left|\hat{\mathscr{E}}_{N+1, y}^{+}(0,0)\right|}{\left|\hat{\mathscr{E}}_{1, y}^{-}(0,0)\right|} .
\end{aligned}
$$

Здесь мы учли, что в силу симметрии задачи $\quad \hat{\mathscr{E}}_{N+1, x}^{+}(0,0)=0, \quad \hat{\mathscr{E}}_{1, x}^{-}(0,0)=0, \quad \hat{\epsilon}_{N+1, y}^{+}(0,0)=0$, $\hat{\epsilon}_{1, y}^{-}(0,0)=0$ (элементарные диполи в точке наблюдения дают отличную от нуля составляющую поля в направлении диполя и нулевую составляющую в перпендикулярном направлении). Заметим, кроме того, что в силу симметрии задачи $\hat{\epsilon}_{1, x}^{-}(0,0)=\hat{\mathscr{E}}_{1, y}^{-}(0,0)$ и $\hat{\epsilon}_{N+1, x}^{+}(0,0)=\hat{\mathscr{E}}_{N+1, y}^{+}(0,0)$.

Так как точки наблюдения находятся в дальней зоне, то и для полных полей будет сохраняться равенство

$$
\frac{\left|E_{N+1}\right|}{\mid E_{1}} \underset{R \rightarrow \infty}{\longrightarrow} \frac{\left|k_{N+1}\right|}{\left|k_{1}\right|} \frac{\left|\hat{\epsilon}_{N+1, x}^{+}(0,0)\right|}{\left|\hat{\epsilon}_{1, x}^{-}(0,0)\right|}=\frac{\left|k_{N+1}\right|}{\left|k_{1}\right|} \frac{\left|\hat{\mathscr{E}}_{N+1, y}^{+}(0,0)\right|}{\left|\hat{\mathscr{E}}_{1, y}^{-}(0,0)\right|} \text {. }
$$

Рассматривая непоглощающие среды, получим, что $\left|k_{N+1}\right| /\left|k_{1}\right|=\sqrt{\varepsilon_{N+1} \mu_{N+1}} / \sqrt{\varepsilon_{1} \mu_{1}}$, а величина

$$
\mathfrak{T}=\frac{\left|\hat{\epsilon}_{N+1, x}^{+}(0,0)\right|}{\left|\hat{\epsilon}_{1, x}^{-}(0,0)\right|}=\frac{\left|\hat{\mathscr{E}}_{N+1, y}^{+}(0,0)\right|}{\left|\hat{\mathscr{E}}_{1, y}^{-}(0,0)\right|}
$$

в общем случае плоскослоистой структуры получается из решения уравнения (30) и, следовательно, является алгебраической функцией толщин пленок, входящих в плоскослоистую структуру, их электрических и магнитных проницаемостей, а также электрических и магнитных проницаемостей окружающих полупространств. Отметим, что из предыдущих рассуждений следует, что если пленки плоскослоистой структуры поглощающие (например, металлические), то предел (54) остается справедливым. Непоглощающими должны быть полупространства, окружающие плоскослоистую структуру, в которых находятся поля. Очевидно, что полученные соотношения легко обобщаются на случай поглощающих полупространств. Если рассматривать поля на больших расстояниях по нормали $R_{1}$ и $R_{N+1}$ в соответствующих 


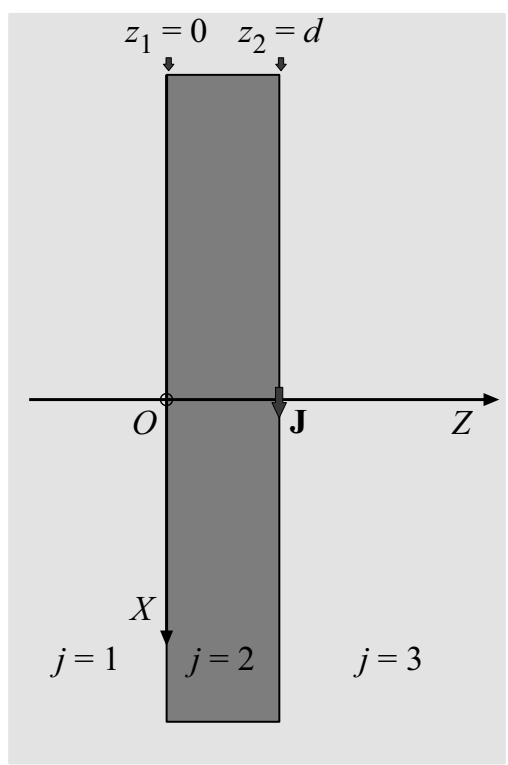

Рис. 5. Геометрия задачи излучения точечного горизонтального электрического диполя на границе пленки.

полупространствах, то формула (54) примет вид при $R_{1} \rightarrow \infty$ и при $R_{N+1} \rightarrow \infty$, где

$$
\frac{\left|E_{N+1}\right|}{E_{1}} \underset{\substack{R_{1} \rightarrow \infty \\ R_{N+1} \rightarrow \infty}}{\longrightarrow} \frac{\left|k_{N+1} e^{i k_{N+1} R_{N+1}}\right|}{\left|k_{1} e^{i k_{1} R_{1}}\right|} \frac{R_{1}}{R_{N+1}} \mathfrak{T},
$$

где $\mathfrak{T}$ определяется (55).

\section{Излучение элементарного горизонтального диполя, расположенного на границе немагнитной пленки}

В качестве конкретного примера, рассмотрим поле горизонтального единичного источника тока, расположенного на свободной границе пленки (рис. 5). Слово „горизонтальный“ мы будем использовать по историческим соображениям, данная задача возникла при рассмотрении горизонтальной дипольной антенны малого размера, расположенного на горизонтальной границе воздуха и земной поверхности. В данном рассмотрении „горизонтальный“ - означает параллельный плоской границе раздела пленка - свободное полупространство.

Будем считать, что поляризация источника - вдоль оси $X\left(n_{x}=1, n_{y}=0, n_{z}=0\right)$. Для нахождения отношения нормально излученных полей по формуле (54) достаточно будет рассмотреть только поляризацию вдоль оси $X$. Толщина пленки равна $d$. Индекс 1 соответствует полупространству материала призмы, 2 - пленке, а 3 свободному полупространству над пленкой. Тогда $N=2$, $z_{d}=z_{2}=d, \mathbf{H}_{R}=\mathbf{T}_{3}, \mathbf{H}_{L}=\left(\mathbf{T}_{1} \times \mathbf{T}_{2}\right)^{-1}$ и уравнение (28) примет вид

$$
\mathbf{T}_{3} \times \hat{\mathfrak{E}}_{3}=\left(\mathbf{T}_{1} \times \mathbf{T}_{2}\right)^{-1} \times \hat{\mathfrak{E}}_{1}+\mathbf{V}
$$

где матрицы выражаются следующими формулами

$$
\begin{gathered}
\mathbf{T}_{1}\left(\begin{array}{cc}
\mathbf{I} & \mathbf{I} \\
\mathbf{G}_{1} & -\mathbf{G}_{1}
\end{array}\right)^{-1}, \\
\mathbf{T}_{2}=\left(\begin{array}{cc}
\mathbf{I} & e^{i \gamma_{2} d} \mathbf{I} \\
\mathbf{G}_{2} & -e^{i \gamma_{2} d} \mathbf{G}_{2}
\end{array}\right)\left(\begin{array}{cc}
e^{i \gamma_{2} d} \mathbf{I} & \mathbf{I} \\
e^{i \gamma_{2} d} \mathbf{G}_{2} & -\mathbf{G}_{2}
\end{array}\right)^{-1}, \\
\mathbf{T}_{3}=\left(\begin{array}{cc}
\mathbf{I} & \mathbf{I} \\
\mathbf{G}_{3} & -\mathbf{G}_{3}
\end{array}\right),
\end{gathered}
$$

а вектор столбец стороннего тока равен $\mathbf{V}=(0 ; 0 ; 0 ;-1)^{T}$.

Учитывая, что $\left(\mathbf{T}_{1} \times \mathbf{T}_{2}\right)^{-1}=\mathbf{T}_{2}^{-1} \times \mathbf{T}_{1}^{-1}$, получим

$$
\begin{aligned}
& \mathbf{H}_{L}=\left(\mathbf{T}_{1} \times \mathbf{T}_{2}\right)^{-1}=\mathbf{T}_{2}^{-1} \times \mathbf{T}_{1}^{-1} \\
& =\left(\begin{array}{cc}
e^{i \gamma_{2} d} \mathbf{I} & \mathbf{I} \\
e^{i \gamma_{2} d} \mathbf{G}_{2} & -\mathbf{G}_{2}
\end{array}\right)\left(\begin{array}{cc}
\mathbf{I} & e^{i \gamma_{2} d} \mathbf{I} \\
\mathbf{G}_{2} & -e^{i \gamma_{2} d} \mathbf{G}_{2}
\end{array}\right)^{-1} \times\left(\begin{array}{cc}
\mathbf{I} & \mathbf{I} \\
\mathbf{G}_{1} & -\mathbf{G}_{1}
\end{array}\right) .
\end{aligned}
$$

Вводя $\quad$ вектор-столбец $\quad \hat{\mathbf{E}}_{\text {out }}=\left(\hat{E}_{1, x}^{-} ; \hat{E}_{1, y}^{-} ; \hat{E}_{3, x}^{+} ; \hat{E}_{3, y}^{+}\right)^{T}$, уравнение (30) для данной задачи примет вид:

$$
\left(\begin{array}{cc}
-\mathbf{H}_{L B} & \mathbf{I} \\
-\mathbf{H}_{L D} & \mathbf{G}_{3}
\end{array}\right) \times \hat{\mathfrak{E}}_{\text {out }}=\mathbf{V} .
$$

Решая линейное уравнение $(57)$, найдем $\hat{E}_{1, x}^{-}, \hat{E}_{1, y}^{-}$и $\hat{E}_{3, x}^{+}, \hat{E}_{3, y}^{+}$, и далее, по формулам (31)-(34) при $N=2$ и получим уходящую волну из плоскослоистой структуры в призму (в область 1) и свободное пространство (область 3) над пленкой (в направлениях $z \rightarrow \pm \infty$ от границ пленки).

Будем считать, что среды немагнитные и $\mu_{1}=\mu_{2}=$ $=\mu_{3}=\mu_{0}$. Тогда, учитывая $k_{j}^{2}=\varepsilon_{j} \mu_{j} \omega^{2}$, выражения для подматриц $\mathbf{H}_{L B}$ и $\mathbf{H}_{L D}$ можно получить в явном виде: подматрица $\mathbf{H}_{L B}$ :

$$
\begin{aligned}
\left(\mathbf{H}_{L B}\right)_{0,0}= & \cos \left(\gamma_{2} d\right)+i\left(\left(\frac{\varepsilon_{1}-\varepsilon_{2}}{\varepsilon_{2}}\right) \frac{\xi^{2}}{\gamma_{1}}-\gamma_{1}\right) \frac{\sin \left(\gamma_{2} d\right)}{\gamma_{2}}, \\
& \left(\mathbf{H}_{L B}\right)_{0,1}=i \xi \eta \frac{\left(\varepsilon_{1}-\varepsilon_{2}\right)}{\varepsilon_{2}} \frac{\sin \left(\gamma_{2} d\right)}{\gamma_{1} \gamma_{2}}, \\
& \left(\mathbf{H}_{L B}\right)_{1,0}=i \xi \eta \frac{\left(\varepsilon_{1}-\varepsilon_{2}\right)}{\varepsilon_{2}} \frac{\sin \left(\gamma_{2} d\right)}{\gamma_{1} \gamma_{2}}, \\
\left(\mathbf{H}_{L B}\right)_{1,1}= & \cos \left(\gamma_{2} d\right)+i\left(\left(\frac{\varepsilon_{1}-\varepsilon_{2}}{\varepsilon_{2}}\right) \frac{\eta^{2}}{\gamma_{1}}-\gamma_{1}\right) \frac{\sin \left(\gamma_{2} d\right)}{\gamma_{2}},
\end{aligned}
$$

подматрица $\mathbf{H}_{L D}$ :

$$
\left(\mathbf{H}_{L D}\right)_{0,0}=\frac{\xi \eta}{\mu_{0} \omega}\left(\frac{\cos \left(\gamma_{2} d\right)}{\gamma_{1}}-i \frac{\sin \left(\gamma_{2} d\right)}{\gamma_{2}}\right),
$$

$$
\begin{aligned}
\left(\mathbf{H}_{L D}\right)_{0,1} & =\frac{\left(\gamma_{1}^{2}+\eta^{2}\right)}{\gamma_{1} \mu_{0} \omega} \cos \left(\gamma_{2} d\right)-i \frac{\left(\gamma_{2}^{2}+\eta^{2}\right)}{\gamma_{2} \mu_{0} \omega} \sin \left(\gamma_{2} d\right), \\
\left(\mathbf{H}_{L D}\right)_{1,0} & =\frac{-\left(\gamma_{1}^{2}+\xi^{2}\right)}{\gamma_{1} \mu_{0} \omega} \cos \left(\gamma_{2} d\right)+i \frac{\left(\gamma_{2}^{2}+\xi^{2}\right)}{\gamma_{2} \mu_{0} \omega} \sin \left(\gamma_{2} d\right),
\end{aligned}
$$




$$
\left(\mathbf{H}_{L D}\right)_{1,1}=-\frac{\xi \eta}{\mu_{0} \omega}\left(\frac{\cos \left(\gamma_{2} d\right)}{\gamma_{1}}-i \frac{\sin \left(\gamma_{2} d\right)}{\gamma_{2}}\right) .
$$

Решив уравнение (57), найдем следующие аналитические выражения для $\hat{E}_{1, x}^{-}, \hat{E}_{1, y}^{-}, \hat{E}_{1, z}^{-}$:

$$
\hat{E}_{1, x}^{-}=\frac{\left(a_{1 x}+d_{1 x} \eta^{2}\right)}{\omega \mu_{0} D} \cos \left(\gamma_{2} d\right)+i \frac{\left(b_{1 x}+e_{1 x} \eta^{2}\right)}{\omega \mu_{0} D} \sin \left(\gamma_{2} d\right),
$$

где

$$
\begin{gathered}
a_{1 x}=-\left(\gamma_{1}+\gamma_{3}\right) ; \quad d_{1 x}=-\left(\gamma_{1}+\gamma_{3}\right) / \gamma_{1} \gamma_{3} ; \\
b_{1 x}=\left(\gamma_{1} \gamma_{3}+\gamma_{2}^{2}\right) / \gamma_{2} ; \quad e_{1 x}=\frac{\left(\gamma_{3}+\gamma_{1}\right)}{\gamma_{2} \gamma_{3}}+\frac{\left(\varepsilon_{2}-\varepsilon_{1}\right) k_{3}^{2}}{\varepsilon_{2} \gamma_{1} \gamma_{2} \gamma_{3}} . \\
\hat{E}_{1, y}^{-}=\frac{1}{\omega \mu_{0} D}\left(a_{1 y} \cos \left(\gamma_{2} d\right)+i b_{1 y} \sin \left(\gamma_{2} d\right)\right) \xi \eta ;
\end{gathered}
$$

где

$$
\begin{gathered}
a_{1 y}=\left(\gamma_{1}+\gamma_{3}\right) / \gamma_{1} \gamma_{3} ; \\
b_{1 y}=\frac{1}{\gamma_{1} \gamma_{2} \gamma_{3}}\left(\frac{\left(\varepsilon_{1}-\varepsilon_{2}\right) k_{3}^{2}}{\varepsilon_{2}}-\gamma_{1}\left(\gamma_{1}+\gamma_{3}\right)\right) . \\
\hat{E}_{1, z}^{-}=\xi \hat{E}_{1, x}^{-} / \gamma_{1}+\eta \hat{E}_{1, y}^{-} / \gamma_{1} ;
\end{gathered}
$$

и $\hat{E}_{3, x}^{+}, \hat{E}_{3, y}^{+}, \hat{E}_{3, z}^{+}$:

$$
\begin{aligned}
\hat{E}_{3, x}^{+} & =\frac{\left(a_{3 x}+d_{3 x} \eta^{2}\right)}{\omega \mu_{0} D} \cos ^{2}\left(\gamma_{2} d\right)+\frac{\left(b_{3 x}+e_{3 x} \eta^{2}\right)}{\omega \mu_{0} D} \sin ^{2}\left(\gamma_{2} D\right) \\
& +i \frac{\left(c_{3 x}+f_{3 x} \eta^{2}\right)}{\omega \mu_{0} D} \cos \left(\gamma_{2} d\right) \sin \left(\gamma_{2} d\right)
\end{aligned}
$$

где

$$
\begin{gathered}
a_{3 x}=-\left(\gamma_{1}+\gamma_{3}\right) \\
d_{3 x}=-\left(\gamma_{1}+\gamma_{3}\right) / \gamma_{1} \gamma_{3} \\
b_{3 x}=\left[\gamma_{1} \gamma_{3}\left(k_{3}^{2}-\gamma_{3}^{2}\right)+\gamma_{3} \gamma_{1}^{3}+\gamma_{2}^{2} k_{1}^{2}\right] /\left(\gamma_{1} k_{2}^{2}\right) \\
e_{3 x}=\left[\gamma_{3} \gamma_{1}^{2}\left(k_{2}^{2}+\gamma_{2}^{2}\right)-\gamma_{3} \gamma_{2}^{4}+k_{1}^{2} \gamma_{1} \gamma_{2}^{2}\right] /\left(k_{2}^{2} \gamma_{1} \gamma_{2}^{2} \gamma_{3}\right) ; \\
c_{3 x}=\frac{\gamma_{3}}{\gamma_{1} \gamma_{2} k_{2}^{2}}\left(\left(\gamma_{2}^{2}+\gamma_{1}^{2}\right)\left(k_{3}^{2}-\gamma_{3}^{2}\right)+2 \gamma_{1}^{2} \gamma_{2}^{2}\right) \\
+\frac{1}{\gamma_{2} k_{2}^{2}}\left(\gamma_{2}^{4}+\gamma_{1}^{2} \gamma_{2}^{2}+2 \gamma_{2}^{2}\left(k_{2}^{2}-\gamma_{2}^{2}\right)\right) ; \\
f_{3 x}=\frac{1}{\gamma_{1} \gamma_{2} \gamma_{3} k_{2}^{2}}\left(k_{3}^{2}\left(\gamma_{2}^{2}+\gamma_{1}^{2}\right)+2 \gamma_{1}^{2} \gamma_{2}^{2}-\gamma_{3}^{2}\left(\gamma_{2}^{2}+\gamma_{1}^{2}\right)\right) \\
+\frac{1}{\gamma_{2} k_{2}^{2}}\left(2 k_{2}^{2}+\gamma_{1}^{2}-\gamma_{2}^{2}\right) . \\
\hat{E}_{3, y}^{+}=\frac{1}{\omega \mu_{0} D}\left(a_{3 y} \cos ^{2}\left(\gamma_{2} d\right)+b_{3 y} \sin ^{2}\left(\gamma_{2} d\right)\right. \\
\left.+i c_{3 y} \cos \left(\gamma_{2} d\right) \sin \left(\gamma_{2} d\right)\right) \xi \eta
\end{gathered}
$$

где

$$
\begin{gathered}
a_{3 y}=\left(\gamma_{1}+\gamma_{3}\right) / \gamma_{1} \gamma_{3} ; \\
b_{3 y}=\frac{\left(\varepsilon_{1}-\varepsilon_{2}\right)}{\varepsilon_{2}} \frac{\left(\gamma_{1}\left(k_{3}^{2}-\gamma_{3}^{2}\right)-\gamma_{2}^{2} \gamma_{3}\right)}{\gamma_{1} \gamma_{2}^{2} \gamma_{3}}-\frac{\gamma_{1}\left(\gamma_{1}+\gamma_{3}\right)}{\gamma_{2}^{2} \gamma_{3}} ;
\end{gathered}
$$

$$
c_{3 y}=\frac{\left(\varepsilon_{1}-\varepsilon_{2}\right)}{\varepsilon_{2}} \frac{\left(k_{3}^{2}-\gamma_{3}\left(\gamma_{1}+\gamma_{3}\right)\right)}{\gamma_{1} \gamma_{2} \gamma_{3}}-\frac{2\left(\gamma_{1}+\gamma_{3}\right)}{\gamma_{2} \gamma_{3}} .
$$

$$
\hat{E}_{3, z}^{+}=-\xi \hat{E}_{3, x}^{+} / \gamma_{3}-\eta \hat{E}_{3, y}^{+} / \gamma_{3} .
$$

В формулах (58)-(63) функция $D(\xi, \eta)$ есть детерминант матрицы уравнения (57), представляющийся следующим аналитическим выражением:

$$
D(\xi, \eta)=\frac{\begin{array}{c}
\Delta_{1}\left(\sqrt{\xi^{2}+\eta^{2}}\right) \cos ^{2}\left(\gamma_{2} d\right)+\Delta_{2}\left(\sqrt{\xi^{2}+\eta^{2}}\right) \sin ^{2}\left(\gamma_{2} d\right)+ \\
+\Delta_{3}\left(\sqrt{\xi^{2}+\eta^{2}}\right) \cos \left(\gamma_{2} d\right) \sin \left(\gamma_{2} d\right)
\end{array}}{\omega^{2} \mu_{0}^{2}}
$$

где

$$
\begin{gathered}
\Delta_{1}(\lambda)=\frac{\left(\gamma_{1}+\gamma_{3}\right)^{2}\left(\gamma_{1} \gamma_{3}+\lambda^{2}\right)}{\gamma_{1} \gamma_{3}} ; \\
\Delta_{2}(\lambda)=-\frac{\left(\gamma_{1} \gamma_{3}+\gamma_{2}^{2}\right)^{2}}{\gamma_{1} \gamma_{3} \gamma_{2}^{2} k_{2}^{2}}\left(\lambda^{4}+\frac{\gamma_{2}^{2}\left(\gamma_{1}+\gamma_{3}\right)^{2}}{\gamma_{1} \gamma_{3}+\gamma_{2}^{2}} \lambda^{2}+\gamma_{1} \gamma_{2}^{2} \gamma_{3}\right) ; \\
\Delta_{3}(\lambda)=-i \frac{\left(\gamma_{1}+\gamma_{3}\right)}{\gamma_{1} \gamma_{2} \gamma_{3} k_{2}^{2}}\left[2\left(\gamma_{1} \gamma_{3}+\gamma_{2}^{2}\right)\left(\gamma_{1} \gamma_{3} \gamma_{2}^{2}+\lambda^{4}\right)\right. \\
\left.+\left(\gamma_{1}^{2} \gamma_{3}^{2}+\gamma_{1}^{2} \gamma_{2}^{2}+4 \gamma_{1} \gamma_{3} \gamma_{2}^{2}+\gamma_{3}^{2} \gamma_{2}^{2}+\gamma_{2}^{4}\right) \lambda^{2}\right] .
\end{gathered}
$$

Естественно, что полученные выражения (58)-(63) переходят в соответствующие выражения для диполя на границе двух полупространств, когда толщина пленки $d$ стремится к нулю.

Аналитические выражения (58)-(63) имеют первостепенное значение, так как позволяют получить выражения для электрических полей в полупространствах через одномерные интегралы, что значительно ускоряет счет и повышает точность вычислений. Действительно, если перейти к полярным координатам в плоскостях $(x, y)$ и $(\xi, \eta)$ по формулам

$x=\rho \cos \varphi, \quad y=\rho \sin \varphi \quad$ и $\quad \xi=\lambda \cos \vartheta, \quad \eta=\lambda \sin \vartheta$,

получим, что величины $\gamma_{1}=\sqrt{k_{1}^{2}-\lambda^{2}}, \gamma_{2}=\sqrt{k_{2}^{2}-\lambda^{2}}$, $\gamma_{3}=\sqrt{k_{3}^{2}-\lambda^{2}}$, выделенные выше множители и детерминант $\mathfrak{D}(\lambda)=D(\lambda \cos \vartheta, \lambda \sin \vartheta)$ являются функциями только $\lambda$ и не зависят от $\vartheta$.

Рассмотрим электрические поля в полупространствах (в областях с номером $j=1$ и $j=3$ ). Сначала рассмотрим (см. представление (31)) в полупространстве c $j=1$.

$$
E_{1, x}(x, y, z)=\frac{1}{(2 \pi)^{2}} \int_{-\infty}^{+\infty} \int_{-\infty}^{+\infty} \hat{E}_{1, x}^{-} e^{-i \gamma_{1} z} e^{i(\xi x+\eta y) d \xi d \eta}
$$


Подставим выражение $(58)$ для $\hat{E}_{1, x}^{-}$и перейдем к полярным координатам (64), получим

$$
\begin{aligned}
& E_{1, x}(\rho, \varphi, z)=\frac{1}{(2 \pi)^{2}} \int_{0}^{+\infty}\left(\int_{0}^{2 \pi}\left(Q_{1 x}(\gamma)+R_{1 x}(\gamma) \lambda^{2} \sin ^{2} \vartheta\right)\right. \\
& \left.\times e^{-i \gamma_{1} z} e^{i \rho \lambda \cos (\varphi-\vartheta)} d \vartheta\right) \lambda d \lambda \\
& =\frac{1}{(2 \pi)^{2}} \int_{0}^{+\infty}\left(Q_{1 x}(\gamma)+R_{1 x}(\gamma) \lambda^{2}\right) \lambda e^{-i \gamma_{1} z} \\
& \times\left(\int_{0}^{2 \pi} e^{i \rho \lambda \cos (\varphi-\vartheta)} d \vartheta\right) d \lambda-\frac{1}{(2 \pi)^{2}} \int_{0}^{+\infty} R_{1 x}(y) \lambda^{3} e^{-i \gamma_{1} z} \\
& \times\left(\int_{0}^{2 \pi} \cos ^{2} \vartheta e^{i \rho \lambda \cos (\varphi-\vartheta)} d \vartheta\right) d \lambda,
\end{aligned}
$$

где введены следующие (зависящие только от $\lambda$ ) функции

$$
\begin{aligned}
& Q_{1 x}(\gamma)=\frac{1}{\omega \mu_{0} \mathfrak{D}(\lambda)}\left(a_{1 x} \cos \left(\gamma_{2} d\right)+i b_{1 x} \sin \left(\gamma_{2} d\right)\right), \\
& R_{1 x}(\gamma)=\frac{1}{\omega \mu_{0} \mathfrak{D}(\lambda)}\left(d_{1 x} \cos \left(\gamma_{2} d\right)+i e_{1 x} \sin \left(\gamma_{2} d\right)\right) .
\end{aligned}
$$

Далее, используя интегральное представление функций Бесселя

$$
J_{n}(\rho \lambda)=\frac{i^{-n}}{2 \pi} \int_{0}^{2 \pi} e^{i \varphi \lambda \cos \theta} e^{i n \theta} d \theta
$$

нетрудно получить следующие представления интегралов

$$
\begin{gathered}
\int_{0}^{2 \pi} e^{i \rho \lambda \cos (\varphi-\theta)} d \vartheta=2 \pi J_{0}(\rho \lambda) \\
\int_{0}^{2 \pi} \cos ^{2} \vartheta e^{i \rho \lambda \cos (\varphi-\vartheta)} d \vartheta=\pi\left(J_{0}(\rho \lambda)-J_{2}(\rho \lambda) \cos 2 \varphi\right) .
\end{gathered}
$$

Подставляя выражения (66), получим

$$
\begin{aligned}
& E_{1, x}(\rho, \varphi, z)=\frac{1}{4 \pi} \int_{0}^{+\infty}\left(2 Q_{1 x}(\gamma)+R_{1 x}(\gamma) \lambda^{2}\right) \lambda e^{-i \gamma_{1} z} \\
& \times J_{0}(\rho \lambda) d \lambda+\frac{\cos 2 \varphi}{4 \pi} \int_{0}^{+\infty} R_{1 x}(\gamma) \lambda^{3} J_{2}(\rho \lambda) e^{-i \gamma_{1} z} d \lambda .
\end{aligned}
$$

Аналогично, для $y$-компоненты. Из (31) и (59) получим из

$$
E_{1, y}(x, y, z)=\frac{1}{(2 \pi)^{2}} \int_{-\infty}^{+\infty} \int_{-\infty}^{+\infty} \hat{E}_{1, y}^{-} e^{-i \gamma_{1} z} e^{i(\xi x+\eta y)} d \xi d \eta
$$

и после перехода к полярным координатам

$$
\begin{aligned}
& E_{1, y}(\rho, \varphi, z)=\frac{1}{(2 \pi)^{2}} \int_{0}^{+\infty}\left(\int_{0}^{2 \pi} Q_{1 y}(\gamma) \lambda^{2} \cos \vartheta \sin \vartheta\right. \\
& \left.\times e^{-i \gamma_{1} z} e^{i \rho \lambda \cos (\varphi-\vartheta)} d \vartheta\right) \lambda d \lambda=\frac{1}{(2 \pi)^{2}} \int_{0}^{+\infty} \lambda^{3} Q_{1 y}(\gamma) e^{-i \gamma_{1} z} \\
& \times\left(\int_{0}^{2 \pi} \cos \vartheta \sin \vartheta e^{i \rho \lambda \cos (\varphi-\vartheta)} d \vartheta\right) d \lambda
\end{aligned}
$$

где введена функция от $\lambda$ :

$$
Q_{1 y}(\lambda)=\frac{1}{\omega \mu_{0} \mathscr{D}(\lambda)}\left(a_{1 y} \cos \left(\gamma_{2} d\right)+i b_{1 y} \sin \left(\gamma_{2} d\right)\right)
$$

Получая из интегрального представления (65) формулу

$$
\begin{aligned}
& \int_{0}^{2 \pi} \sin \vartheta \cos \vartheta e^{i \rho \lambda \cos (\varphi-\vartheta)} d \vartheta \\
& =\pi \sin 2 \varphi\left(J_{0}(\rho \lambda)-\frac{2}{\rho \lambda} J_{1}(\rho \lambda)\right),
\end{aligned}
$$

получим для $E_{1, y}$ выражение

$$
\begin{aligned}
& E_{1, y}(\rho, \varphi, z)=\frac{\sin 2 \varphi}{4 \pi} \int_{0}^{+\infty} \lambda^{3} Q_{1 y}(\gamma) \\
& \times\left(J_{0}(\rho \lambda)-\frac{2}{\rho \lambda} J_{1}(\rho \lambda)\right) e^{-i \gamma_{1} z} d \lambda
\end{aligned}
$$

Оставшуюся компоненту $E_{1, z}$ в первом полупространстве из (31) запишем в виде

$$
\begin{aligned}
E_{1, z}(x, y, z) & =\frac{1}{(2 \pi)^{2}} \int_{-\infty}^{+\infty} \int_{-\infty}^{+\infty}\left(\xi \hat{E}_{1, x}^{-} / \gamma_{1}+\eta \hat{E}_{1, y}^{-} / \gamma_{1}\right) \\
& \times e^{-i \gamma_{1} z} e^{i(\xi x+\eta y)} d \xi d \eta
\end{aligned}
$$

и, подставляя выражения для $\hat{E}_{1, x}^{-}$и $\hat{E}_{1, y}^{-}$, по формулам (58) и (59), после перехода к полярным координатам, 
получим

$$
\begin{aligned}
& E_{1, z}(\rho, \varphi, z)=\frac{1}{(2 \pi)^{2}} \int_{0}^{+\infty} \frac{Q_{1 x}(\gamma) \lambda^{2} e^{i \gamma_{1} z}}{\gamma_{1}} \\
& \times\left(\int_{0}^{2 \pi} \cos \vartheta e^{i \rho \lambda \cos (\varphi-\vartheta)} d \vartheta\right) d \lambda \\
& +\frac{1}{(2 \pi)^{2}} \int_{0}^{+\infty} \frac{\left.R_{1 x}(\gamma)+Q_{1 y}(\gamma)\right) \lambda^{4}}{\gamma_{1}} e^{i \gamma_{1} z} \\
& \times\left(\int_{0}^{2 \pi} \cos \vartheta \sin ^{2} \vartheta e^{i \rho \lambda \cos (\varphi-\vartheta)} d \vartheta\right) d \lambda .
\end{aligned}
$$

Получая из представления (65) выражения

$$
\begin{aligned}
& \int_{0}^{2 \pi} \cos \vartheta e^{i \rho \lambda \cos (\varphi-\vartheta)} d \vartheta=2 \pi i \cos \varphi J_{1}(\rho \lambda), \\
& \int_{0}^{2 \pi} \cos \vartheta \sin ^{2} \vartheta e^{i \rho \lambda \cos (\varphi-\vartheta)} d \vartheta \\
& =i 2 \pi \cos \varphi\left(\sin ^{2} \varphi J_{1}(\rho \lambda)+\left(1-4 \sin ^{2} \varphi\right) \frac{J_{2}(\rho \lambda)}{\rho \lambda}\right),
\end{aligned}
$$

получим

$$
\begin{aligned}
& E_{1, z}(\rho, \varphi, z)=\frac{i \cos \varphi}{2 \pi} \int_{0}^{+\infty} \frac{Q_{1 x}(\gamma) \lambda^{2} e^{-i \gamma_{1} z}}{\gamma_{1}} J_{1}(\rho \lambda) d \lambda \\
& +\frac{i \cos \varphi \sin ^{2} \varphi}{2 \pi} \int_{0}^{+\infty} \frac{\left(R_{1 x}(\gamma)+Q_{1 y}(\gamma)\right) \lambda^{4} J_{1}(\rho \lambda)}{\gamma_{1}} e^{-i \gamma_{1} z} d \lambda \\
& +\frac{i \cos \varphi\left(1-4 \sin ^{2} \varphi\right)}{2 \pi} \int_{0}^{+\infty} \frac{\left(R_{1 x}(\gamma)+Q_{1 y}(\gamma)\right) \lambda^{3} J_{2}(\rho \lambda)}{\rho \gamma_{1}} e^{-i \gamma_{1} z} d \lambda .
\end{aligned}
$$

Теперь рассмотрим электрическое поле в полупространстве (с номером $j=3$ ). Составляющую по оси $x$ получим из (33) в виде

$$
E_{3, x}(x, y, z)=\frac{1}{(2 \pi)^{2}} \int_{-\infty}^{+\infty} \int_{-\infty}^{+\infty} \hat{E}_{3, x}^{+} e^{i \gamma_{3}(z-d)} e^{i(\xi x+\eta y)} d \xi d \eta
$$

Преобразуя выражение (61), его можно представить в виде

$$
\begin{aligned}
& \hat{E}_{3, x}^{+}=\frac{1}{\omega \mu_{0} D}\left[a_{3 x} \cos ^{2}\left(\gamma_{2} d\right)+b_{3 x} \sin ^{2}\left(\gamma_{2} d\right)\right. \\
& +i c_{3 x}\left(\gamma_{2} d\right) \cos \left(\gamma_{2} d\right) \sin \left(\gamma_{2} d\right)+\left(d_{3 x} \cos ^{2}\left(\gamma_{2} d\right)\right. \\
& \left.\left.+e_{3 x} \sin ^{2}\left(\gamma_{2} d\right)+i f_{3 x} \cos \left(\gamma_{2} d\right) \sin \left(\gamma_{2} d\right)\right) \eta^{2}\right] .
\end{aligned}
$$

Тогда, переходя к полярным координатам (64), получим

$$
\begin{aligned}
& E_{3, x}(\rho, \varphi, z)=\frac{1}{(2 \pi)^{2}} \int_{0}^{+\infty}\left(\int_{0}^{2 \pi}\left(Q_{3 x}(\gamma)+R_{3 x}(\gamma) \lambda^{2} \sin ^{2} \vartheta\right)\right. \\
& \left.\times e^{i \gamma_{3}(z-d)} e^{i \rho \lambda \cos (\varphi-\vartheta)} d \vartheta\right) \lambda d \lambda \\
& =\frac{1}{(2 \pi)^{2}} \int_{0}^{+\infty}\left(Q_{3 x}(\gamma)+\lambda^{2} R_{3 x}(\gamma)\right) e^{i \gamma_{3}(z-d)} \\
& \times e^{i \rho \lambda \cos (\varphi-\vartheta)}\left(\int_{0}^{2 \pi} e^{i \rho \lambda \cos (\varphi-\vartheta)} d \vartheta\right) \lambda d \lambda \\
& \left.-\frac{1}{(2 \pi)^{2}} \int_{0}^{+\infty} \lambda^{2} R_{3 x}(\gamma)\right) e^{i \gamma_{3}(z-d)}\left(\int_{0}^{2 \pi} \cos ^{2} \vartheta e^{i \rho \lambda \cos (\varphi-\vartheta)} d \vartheta\right) \lambda d \lambda,
\end{aligned}
$$

где введены следующие, зависящие только от $\lambda$, функции

$$
\begin{aligned}
& Q_{3 x}(\gamma)=\frac{1}{\omega \mu_{0} \mathscr{D}(\lambda)}\left(a_{3 x} \cos ^{2}\left(\gamma_{2} d\right)\right. \\
& \left.+b_{3 x} \sin ^{2}\left(\gamma_{2} d\right)+i c_{3 x} \cos \left(\gamma_{2} d\right) \sin \left(\gamma_{2} d\right)\right), \\
& R_{3 x}(\gamma)=\frac{1}{\omega \mu_{0} \mathscr{D}(\lambda)}\left(d_{3 x} \cos ^{2}\left(\gamma_{2} d\right)\right. \\
& \left.+e_{3 x} \sin ^{2}\left(\gamma_{2} d\right)+i f_{3 x} \cos \left(\gamma_{2} d\right) \sin \left(\gamma_{2} d\right)\right) .
\end{aligned}
$$

Подставляя выражения (66), получим

$$
\begin{aligned}
& E_{3, x}(\rho, \varphi, z)=\frac{1}{4 \pi} \int_{0}^{+\infty}\left(2 Q_{3 x}(\gamma)+\lambda^{2} R_{3 x}(\gamma)\right) J_{0}(\rho \lambda) \\
& \times e^{i \gamma_{3}(z-d)} \lambda d \lambda+\frac{\cos 2 \varphi}{4 \pi} \int_{0}^{+\infty} \lambda^{3} R_{3 x}(\gamma) J_{2}(\rho \lambda) e^{i \gamma_{3}(z-d)} d \lambda .
\end{aligned}
$$

Аналогично,, в $\quad$ представлении $\quad E_{3 y}(x, y, z)=$ $=(2 \pi)^{-2} \int_{-\infty}^{+\infty} \int_{-\infty}^{+\infty} \hat{E}_{3, y}^{+} e^{i \gamma_{3}(z-d)} e^{i(\xi x+\eta y)} d \xi d \eta \quad$ запишем $\hat{E}_{3, y}^{+}$в виде (62) и перейдем к полярным координатам

$$
E_{3, y}(\rho, \varphi, z)=\frac{1}{(2 \pi)^{2}} \int_{0}^{+\infty}\left(\int_{0}^{2 \pi} Q_{3 y}(\gamma) \lambda^{2} \cos \vartheta \sin \vartheta\right.
$$

$$
\begin{aligned}
& \left.\times e^{i \gamma_{3}(z-d)} e^{i \rho \lambda(\varphi-\vartheta)} d \vartheta\right) \lambda d \lambda=\frac{1}{(2 \pi)^{2}} \int_{0}^{+\infty} \lambda^{3} Q_{3 y}(\gamma) e^{i \gamma_{3}(z-d)} \\
& \times\left(\int_{0}^{2 \pi} \cos \vartheta \sin \vartheta e^{i \rho \lambda(\varphi-\vartheta)} d \vartheta\right) d \lambda,
\end{aligned}
$$


где $\quad Q_{3 y}(\gamma)=\frac{1}{\omega \mu_{0} \mathscr{D}(\lambda)}\left(a_{3 y} \cos ^{2}\left(\gamma_{2} d\right)+b_{3 y} \sin ^{2}\left(\gamma_{2} d\right)+\right.$ $\left.+i c_{3 y} \cos \left(\gamma_{2} d\right) \sin \left(\gamma_{2} d\right)\right)$. Учитывая выражения для интеграла (68), получим

$$
\begin{aligned}
& E_{3, y}(\rho, \varphi, z)=\frac{\sin 2 \varphi}{4 \pi} \int_{0}^{+\infty} \lambda^{3} Q_{3 y}(\gamma) \\
& \times\left(J_{0}\left(\rho \lambda-\frac{2}{\rho \lambda} J_{1}(\rho \lambda)\right)\right) e^{i \gamma_{3}(z-d)} d \lambda .
\end{aligned}
$$

Наконец, для составляющей $E_{3, z}$, учитывая (63), получим аналогично

$$
\begin{aligned}
& E_{3, z}(x, y, z)=-\frac{1}{(2 \pi)^{2}} \int_{-\infty}^{+\infty} \int_{-\infty}^{+\infty}\left(\frac{\xi \hat{E}_{3, x}^{+}}{\gamma_{3}}+\frac{\eta \hat{E}_{3, y}^{+}}{\gamma_{3}}\right) \\
& \times e^{i \gamma_{3}(z-d)} e^{i(\xi x+\eta y)} d \xi d \eta
\end{aligned}
$$

Переходя к полярным координатам, получим следующее выражение

$$
\begin{aligned}
& E_{3, z}(\rho, \varphi, z)=-\frac{1}{(2 \pi)^{2}} \int_{0}^{+\infty} \frac{Q_{3 x}(\gamma) \lambda^{2} e^{i \gamma_{3}(z-d)}}{\gamma_{3}} \\
& \times\left(\int_{0}^{2 \pi} \cos \vartheta e^{i \rho \lambda(\varphi-\vartheta)} d \vartheta\right) d \lambda-\frac{1}{(2 \pi)^{2}} \int_{0}^{+\infty} \frac{\left(R_{3 x}(\gamma)+Q_{3 y}(\gamma)\right) \lambda^{4}}{\gamma_{3}} \\
& \times e^{i \gamma_{3}(z-d)}\left(\int_{0}^{2 \pi} \cos \vartheta e^{i \rho \lambda(\varphi-\vartheta)} d \vartheta\right) d \lambda .
\end{aligned}
$$

Учитывая (70) и (71), получим

$$
\begin{gathered}
E_{3, z}(\rho, \varphi, z)=-\frac{i \cos \varphi}{2 \pi} \int_{0}^{+\infty} \frac{Q_{3 x}(\gamma) \lambda^{2} J_{1}() \rho \lambda}{\gamma_{3}} e^{i \gamma_{3}(z-d)} d \lambda \\
-\frac{i \cos \varphi \sin ^{2} \varphi}{2 \pi} \int_{0}^{+\infty} \frac{\left(R_{3 x}(\gamma)+Q_{3 x}(\gamma)\right) \lambda^{4} J_{1}(\rho \lambda)}{\gamma_{3}} e^{i \gamma_{3}(z-d)} d \lambda \\
-\frac{i \cos \varphi\left(1-4 \sin ^{2} \varphi\right)}{2 \pi} \int_{0}^{+\infty} \frac{\left(R_{3 x}(\gamma)+Q_{3 y}(\gamma)\right) \lambda^{3} J_{2}(\rho \lambda)}{\rho \gamma_{3}} e^{i \gamma_{3}(z-d)} d \lambda .
\end{gathered}
$$

Таким образом, мы получили точные аналитические выражения для электрических полей от элементарного излучателя, расположенного на границе пленки, в полупространствах, окружающих пленку.

\section{Излучение по нормали произвольного поверхностного распределения сторонних токов на границе пленки в дальней зоне}

Теперь мы можем перейти к вычислению отношению полей произвольного поверхностного распределения сторонних токов, излученных по нормали к поверхности пленки. Замечая, что по формулам (58)-(63) мы вычислили $\hat{\epsilon}_{1, x}^{-}(\xi, \eta), \hat{\epsilon}_{1, y}^{-}(\xi, \eta), \hat{\epsilon}_{3, x}^{+}(\xi, \eta), \hat{\epsilon}_{3, y}^{+}(\xi, \eta)$, мы можем найти отношение $\mathfrak{T}=\left|\hat{\epsilon}_{3, x}^{+}(0,0)\right| /\left|\hat{\epsilon}_{1, x}^{-}(0,0)\right|$ и по формуле (54) отношение модулей (амплитуд) электрических полей, излученных произвольным поверхностным током, на одном и том же расстоянии от него, в дальней зоне. Тогда, подставляя в (58) и (61) $\xi 0$ и $\eta=0$, получим

$$
\begin{aligned}
& \hat{\epsilon}_{1, x}^{-}(0,0)=\left.\hat{E}_{1, x}^{-}\right|_{\substack{\xi=0 \\
\eta=0}}=\frac{1}{\omega \mu_{0} D(0,0)}\left[-\left(k_{1}+k_{3}\right) \cos \left(k_{2} d\right)\right. \\
& \left.+i\left(k_{2}+\left(k_{1} k_{3}\right) / k_{2}\right) \sin \left(k_{2} d\right)\right], \\
& \quad \hat{\epsilon}_{3, x}^{+}(0,0)=\left.\hat{E}_{3, x}^{+}\right|_{\substack{\xi=0 \\
\eta=0}}=\frac{1}{\omega \mu_{0} D(0,0)}\left[-\left(k_{1}+k_{3}\right)\right. \\
& \quad \times \cos ^{2}\left(k_{2} d\right)+\frac{k_{1}\left(\left(k_{3} k_{1}\right)+k_{2}^{2}\right)}{k_{2}^{2}} \sin ^{2}\left(k_{2} d\right) \\
& \left.+i \frac{2 k_{3} k_{1}+k_{2}^{2}+k_{1}^{2}}{k_{2}} \cos \left(k_{2} d\right) \sin \left(k_{2} d\right)\right],
\end{aligned}
$$

и окончательно, по формуле (54), получим

$$
\frac{\left|E_{3}\right|}{\left|E_{1}\right|} \underset{R \rightarrow \infty}{\longrightarrow} \frac{k_{3}}{k_{1}} \mathfrak{T}=\frac{\sqrt{\varepsilon_{3}}}{\sqrt{\varepsilon_{1}}} \mathfrak{T},
$$

где

$$
\mathfrak{T}=\frac{\mid-\cos ^{2}\left(k_{2} d\right)+\frac{k_{1}\left(k_{3} k_{1}+k_{2}^{2}\right)}{k_{2}^{2}\left(k_{1}+k_{3}\right)} \sin ^{2}\left(k_{2} d\right)}{-\cos \left(k_{2} d\right)+i \frac{k_{1}^{2}+k_{2}^{2}+2 k_{3} k_{1}}{k_{2}\left(k_{1}+k_{3}\right)} \cos \left(k_{2} d\right) \sin \left(k_{2} d\right) \mid} .
$$

Зная отношение амплитуд полей, легко найти отношение интенсивностей. Так как $I_{i} \sim\left|E_{i}\right|^{2} / w_{i}$, где $w_{i}=\sqrt{\mu_{i} \varepsilon_{i}}-$ волновое сопротивление $i$-го полупространства, то, учитывая $\mu_{1}=\mu_{2}=\mu_{3}=\mu_{0}$, из (76) получим при $R \rightarrow \infty$

$$
\begin{aligned}
& \frac{I_{3}}{I_{1}} \rightarrow\left(\frac{k_{3}}{k_{1}}\right)^{2} \mathfrak{T}^{2} \frac{w_{1}}{w_{3}} \\
& =\left(\frac{\varepsilon_{3}}{\varepsilon_{1}}\right)^{\frac{3}{2}}\left(\frac{\mu_{1}}{\mu_{3}}\right)^{\frac{1}{2}} \mathfrak{T}^{2}=\left(\frac{\varepsilon_{3}}{\varepsilon_{1}}\right)^{\frac{3}{2}} \mathfrak{T}^{2},
\end{aligned}
$$

Как нетрудно убедиться, функция (77), при уменьшении толщины пленки $d$ к нулю или при приближении параметров пленки к параметрам полупространства 


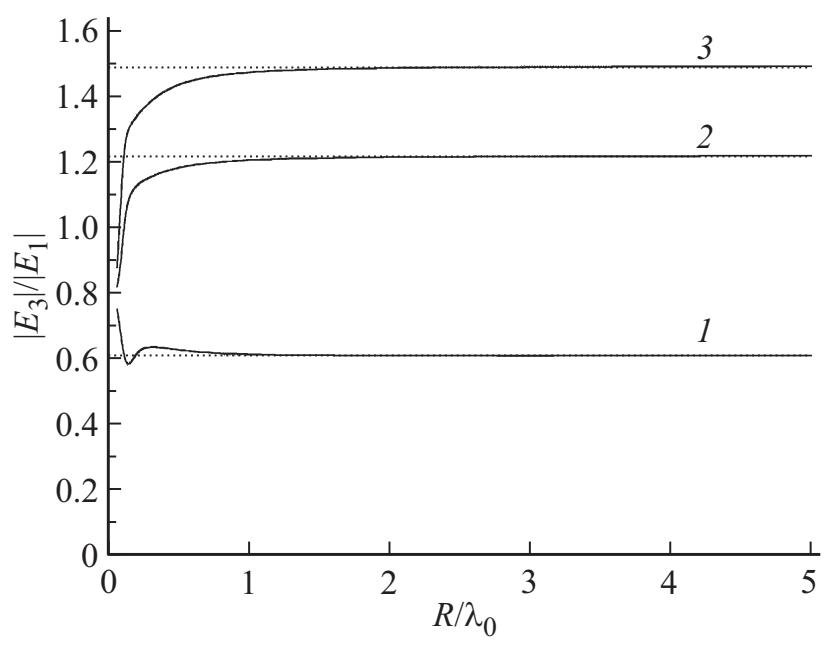

Pис. 6. $\left|E_{3}\right| /\left|E_{1}\right|$ как функции $R / \lambda_{0}$ (по нормали) для горизонтального точечного горизонтального электрического диполя на границе пленки с $\varepsilon_{2}=3 \varepsilon_{0}$ толщиной $d=0.1 \lambda_{0}$, окруженной двумя непоглощающими и немагнитными полупространствами с $\varepsilon_{1}=2 \varepsilon_{0}$ и разными $\varepsilon_{3}$. Кривая 1 соответствует отношению $\varepsilon_{3} / \varepsilon_{1}$ равному 0.5 ; кривая $2-\varepsilon_{3} / \varepsilon_{1}=2$; кривая $3-\varepsilon_{3} / \varepsilon_{1}=3$. Пунктиром показаны асимптоты.

$k_{2} \rightarrow k_{3}$, стремится к единице. Поэтому для произвольного поверхностного распределения сторонних токов (произвольной поверхностной антенны) на границе непоглощающих (с диэлектрическими проницаемостями $\varepsilon_{1}$ и $\varepsilon_{3}$ ) и немагнитных (с магнитными проницаемостями $\left.\mu_{1}=\mu_{3}=\mu_{0}\right)$ полупространств, из (76) и (78), получим при $R \rightarrow \infty$

$$
\frac{\left|E_{3}\right|}{\left|E_{1}\right|} \rightarrow \frac{\sqrt{\varepsilon_{3}}}{\sqrt{\varepsilon_{1}}} \text { и } \frac{I_{3}}{I_{1}} \rightarrow\left(\frac{\varepsilon_{3}}{\varepsilon_{1}}\right)^{\frac{3}{2}}
$$

Отметим, что формула (77), вообще говоря, справедлива и для поглощающей немагнитной пленки и непоглощающих немагнитных полупространств.

Чтобы оценить, как быстро при $R \rightarrow \infty$ отношения полей выходят на константу (76), рассмотрим зависимость от $R$ для точного отношения полей $\left|E_{3}\right| /\left|E_{1}\right|$ от элементарного горизонтально диполя на пленке (малой горизонтальной антенны), которые вычисляются по точным формулам (67) и (73).

На рис. 6 показаны точные зависимости $\left|E_{3}\right| /\left|E_{1}\right|$, как функции $R / \lambda_{0}$ для горизонтального точечного электрического диполя, расположенного на границе пленки диэлектрика толщиной $d=0.1 \lambda_{0}$ и проницаемостью $\varepsilon_{2}=3 \varepsilon_{0}$, окруженной двумя непоглощающими и немагнитными полупространствами с $\varepsilon_{1}=2 \varepsilon_{0}$ и разными $\varepsilon_{3}$. Здесь введены обозначения: $\lambda_{0}$ - длина волны в вакууме, $R$ - расстояние от диполя до точек наблюдения по нормали к границе раздела. На рис. 6 показаны зависимости для нескольких значений $\varepsilon_{3} / \varepsilon_{1}: 0.5,2$ и 3 (кривые 1, 2 и 3, соответственно). Видно, что при росте нормированного расстояния $R / \lambda_{0}$ от источника, кривые быстро приближаются к асимптотам, вычисленным по формуле (76), (77). Асимптоты показаны на рис. 6 пунктиром.

\section{Заключение}

Итак, предложено теоретическое рассмотрение электромагнитного излучения плоских поверхностных токов (плоской антенны), расположенной на границе свободного полупространства и плоскослоистой среды. Найдена фундаментальная закономерность излучения такого источника по нормали к границе раздела. Подробно рассмотрены два частных случая: когда излучающие поверхностные токи, расположены на границе одной пленки и когда эти токи расположены на границе двух однородных полупространств. Отметим, что аналогичная закономерность деления излучения в нормальном направлении для щелевой антенны, вырезанной в тонком проводящем экране, расположенном на границе двух полупространств, была обнаружена более 30 лет назад [24]. Однако, теоретические методы, примененные в работе [24], не позволили обобщить результат на тонкие поверхностные вибраторные антенны. Данная работа демонстрирует, что это обобщение существует и его можно доказать строго, в широкой теоретической формулировке.

\section{Список литературы}

[1] Sommerfeld A. // Ann. Physik. (Leipzig). 1926. V. 81. P. 1135.

[2] Wait J.R. // IEEE Antennas and Propagation Magazine. 1998. V. 40. N 5. P. 7.

[3] King R.W.P., Smith G.S. Antennas in Matter. Cambridge, MA: M.I.T. Press, 1981.

[4] Wu T.T. // J. Appl. Phys. 1957. V. 28. N 3. P. 299.

[5] Jansen R.H. // IEEE Trans. Microwave Theory Tech. 1985. V. 33. N 10. P. 1043.

[6] King R.W.P. // IEEE Trans. Microwave Theory Tech. 1988. V. 36. N 6. P. 1080.

[7] Новотный Л., Хехт Б. Основы нанооптики. Пер. с англ. Под ред. Самарцева В.В. М.: Физматлит, 2009.

[8] Pendry J.B. // Phys. Rev. Let. 2000. V. 85. N 18. P. 3966.

[9] Петрин А.Б. // Оптика и спектроскопия. 2020. Т. 128. № 11. C. 1676.

[10] Петрин А.Б. // ЖЭТФ. 2021. Т. 159. В. 1. С. 35.

[11] Петрин А.Б. // Оптика и спектроскопия. 2021. Т. 129. № 1. C. 55.

[12] Петрин А.Б. // Оптика и спектроскопия. 2020. Т. 128. № 12. C. 1874.

[13] Bharadwaj P., Deutsch B., Novotny L. // Adv. Opt. Photonics. 2009. V. 1. P. 438.

[14] Yu N., Cubukcu E., Diehl L., et al. // Opt. Express. 2007. V. 15. V. 20. P. 13272.

[15] Khaleque A., Mironov E.G., Osório J.H., et al. // Opt. Express. 2017. V. 25. N 8. P. 8986

[16] Петрин А.Б. // ЖЭТФ. 2008. Т. 134. № 3(9). С. 436.

[17] Петрин А.Б. // Оптика и спектроскопия. 2018. Т. 125. № 3. C. 375 . 
[18] Петрин А.Б. // Оптика и спектроскопия. 2019. Т. 126. № 3. C. 350.

[19] Петрин А.Б. // Оптика и спектроскопия. 2019. Т. 127. № 4. C. 654.

[20] Стрэттон Джс.А. Теория электромагнетизма. Пер. с англ. Москва-Ленинград, 1948.

[21] Stratton J.A., Chu L.J. // Phys. Rev. 1939. V. 56. P. 99.

[22] Schelkunoff S.A. // Phys. Rev. 1939. V. 56. P. 308.

[23] Harrington R.F. Ti-Harmonic Electromagnetic Fields. New York: McGraw-Hill, 1961.

[24] Петрин А.Б. Исследование щелевых антенн. Дисс. канд. физ.-матем. наук, Москва, МФТИ, 1988. 